\title{
Juraj Virsik
}

A generalized point of view to higher order connections on fibre bundles

Czechoslovak Mathematical Journal, Vol. 19 (1969), No. 1, 110-142

Persistent URL: http://dml.cz/dmlcz/100882

\section{Terms of use:}

(C) Institute of Mathematics AS CR, 1969

Institute of Mathematics of the Czech Academy of Sciences provides access to digitized documents strictly for personal use. Each copy of any part of this document must contain these Terms of use.

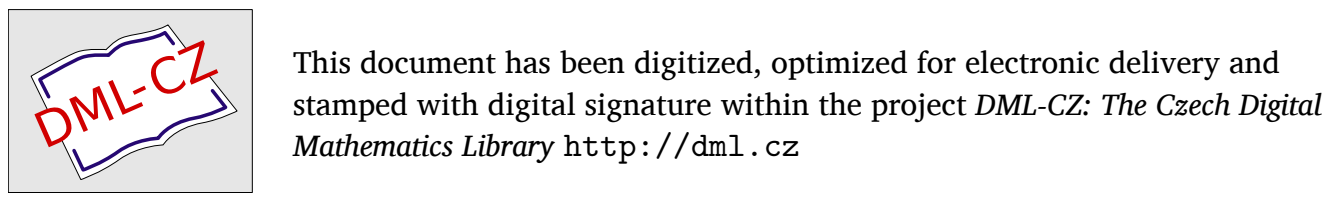




\title{
A GENERALIZED POINT OF VIEW TO HIGHER ORDER CONNECTIONS ON FIBRE BUNDLES
}

\author{
JURAJ VIRSIK, Bratislava \\ (Received October 5, 1967)
}

Higher order connections in differentiable fibre bundles, as introduced and studied by $\mathrm{CH}$. EHRESMANN in his famous note [5] as well as in his unpublished lectures, have been dealt with in the most general form, as to the author's knowledge, only in the papers of B. CenkL ([1], [2]), and, to some extend, by P. Libermann in [12], and by P. Ver EeCKe in [17]. However, the last two papers are mostly concerned with more special situations (e.g. that of a vector bundle) as well as several other ones (c.f. [9], [10], [11], [13], [15], [16], [18], [19]).

Therefore the first two chapters of this paper are supposed to serve as an introduction to the Ehresmann's approach to the very definition of a higher order connection in a differentiable groupoid. Nevertheless the main purpose is to introduce the notion of a $(\mathscr{V}, \mathscr{T})$-connection on a differentiable fibre bundle $E$ (i.e. in its associated groupoid) together with some equivalent formulations. The pair $(\mathscr{V}, \mathscr{T})$ is defined by a subcategory $\mathscr{V}$ of the category $\mathscr{E}$ of all fibered manifolds $(B, C)\left(p_{B}=p: C \rightarrow B\right.$ being the natural projection), and by a covariant functor $\mathscr{T}$ (called connector) from the category of all $C^{\infty}$-differentiable manifolds into $\mathscr{V}$ satisfying some additional assumptions (c.f. definition 3.1). A ( $\mathscr{V}, \mathscr{T})$-connection in a locally trivial differentiable groupoid $\Phi^{\bullet}$ is now given by a mapping $\mathscr{X}: \mathscr{T}(B) \rightarrow \mathscr{T}(\Phi)$ such that $(\sim, \mathscr{X}) \in \mathscr{V}$, $\mathscr{T}(b) \mathscr{X}=\operatorname{id}_{\mathscr{T}(B)}$ and $\mathscr{T}(a) \mathscr{X}=\iota p$, where $\sim: B \rightarrow \Phi$ is the natural injection of the submanifold of unities (objects) into $\Phi, a$ or $b$ are the right and left unit projections respectively from $\Phi$ onto $B$, and $\iota=\iota_{B}$ is a canonical injection $B \rightarrow \mathscr{T}(B)$ (c.f. definition 3.3). A higher order connection in the sense of Ehresmann is a special case of a $(\mathscr{V}, \mathscr{T})$-connection, and in this way one obtains in a unified and formalized manner the various characterizations of higher order connections given in [1], [2] and also sketched in [12]. However, the definition of a semi-holonomic higher order connection given in [12] is more general than that of Ehresmann (except of order one) but it is included in the definition of a $(\mathscr{V}, \mathscr{T})$-connection (c.f. theorem 3.2) with $\mathscr{V}$ being the category of vector bundles and $\mathscr{T}$ the functor $\bar{T}_{r, 1}$ connected with semi-holonomic tangent vectors of order $r \geqq 1$. On the other hand, although each higher order con- 
nection in $\Phi^{\bullet}$ induces a differentiable lifting of the corresponding manifold of velocities in $B$ into the corresponding manifold of infinitesimal displacements of fibres in $E$ (c.f. [5]), this does not hold conversely unless the higher order connection is replaced by a $(\mathscr{V}, \mathscr{T})$-connection with $\mathscr{V}=\mathscr{E}$ and suitable $\mathscr{T}$, which is more general than an Ehresmann's connection. This is namely the analogous situation to that in [12] if one, roughly said, replaces the vectors by velocities and drops the vector bundle structures in them even if the order is one.

This impossibility of defining a higher order connection as a simple lifting either of velocities or of vectors, which complicates also the "associated principal bundle formulations" given in [1] and [2] and which does not occur in the first order theory led to our generalization of a higher order connection to a $(\mathscr{V}, \mathscr{T})$-connection. The other reason may be found in the already mentioned unified formalism including both the "velocity and vector points of view". Moreover the most abstract form of a $(\mathscr{V}, \mathscr{T})$-connection as given in definition 3.1 may suggest a formally easy generalization of the notion of a connection (without pointing out explicitly the order) to more general structures than that of differentiability and with finite dimension. However, we do not touch that problem in this paper.

On the other hand the pair $(\mathscr{V}, \mathscr{T})$ is essential in the definition of a $(\mathscr{V}, \mathscr{T})$ connection unless it is a posteriori clear that it is equivalent to some type of an Ehresmann's connection. Thus a $(\mathscr{V}, \mathscr{T})$-connection is de facto not a property of the groupoid $\Phi^{\bullet}$ itself, but rather a relation of the groupoid $\Phi^{\bullet}$ to the pair $(\mathscr{V}, \mathscr{T})$. In general namely there cannot be found a reasonable definition of the equivalence of two $(\mathscr{V}, \mathscr{T})$-connections unless they are, of course, equivalent to an Ehresmann's one. Therefore, as a matter of fact, the notion of a $(\mathscr{V}, \mathscr{T})$-connection does not generalize the notion of a connection itself but more likely the various "lifting properties" of connections. Nevertheless the formalism employed hereby, namely that connected with $\mathscr{T}$, generalizes and even simplified some classical notations from the first order differential geometry (c.f. ch. 3).

The third chapter is readable formally independently - but for some almost evident notations - from the first two chapters which are of an introductory and illustrating character. Hereby I'd like to express my thanks to O. KowALSKI and I. Kolář for their discussions concerning the first chapter at the seminar on global differential geometry in Brno.

\section{SOME REMARKS ON DIFFERENTIABLE GROUPOIDS}

Definition 1.1. ([6]) A category $C^{*}$ is a class $C$ of elements in which there is given a composition $(X, Y) \rightarrow X$. $Y$, for some pairs of elements in $C$, satisfying the following three axioms:

1. If either $X .(Y . Z)$ or $(X . Y) . Z$ are defined then both they are defined and equal.

2. If $X . Y$ and $Y . Z$ are defined then also $X .(Y . Z)$ is defined. 
3. For each $X \in C$ there are two unities $a X$ and $b X$ in $C$ such that $X$. $a X$ and $b X . X$ are defined. Hereby an element $e \in C$ is called a unity if $X . e=X$ and $e . Y=Y$ for all elements in $C$ for which $X . e$ and $e . Y$ are defined.

These axioms imply: The unities $a X$ and $b X$ are uniquely determined by $X, X . Y$ is defined iff $a X=b Y$, and $a(X . Y)=a Y, b(X . Y)=b X$. The elements of $C$ are called morphisms, the unities among them are called objects. Thus we identify each class with the identity map of this class and write $X \in C, X: a X \rightarrow b X$. A subclass $C^{\prime} \subset C$ is called a subcategory $C^{\prime \bullet}$ of $C^{\bullet}$ if $\left.\mathbf{1}\right) X \in C^{\prime}, Y \in C^{\prime}$ and $X . Y$ is defined in $C^{\bullet}$ implies $X . Y \in C^{\prime}$; 2) $X \in C^{\prime}$ implies $a X \in C^{\prime}$ and $b X \in C^{\prime}$. The subcategory $C^{\prime \bullet}$ is called a complete subcategory of $C^{*}$ if moreover $X \in C, a X \in C^{\prime}$ and $b X \in C^{\prime}$ imply $X \in C^{\prime}$. If now $C^{\bullet}$ and $C^{\prime}$ are two arbitrary categories then a mapping $\mathscr{T}: C \rightarrow C^{\prime}$ satisfying 1) if $X \in C, Y \in C$ and $X . Y$ is defined then $\mathscr{T}(X . Y)=\mathscr{T}(X) . \mathscr{T}(Y)$; 2) if $e$ is a unity in $C^{\circ}$ then $\mathscr{T}(e)$ is a unity in $C^{\prime}$, is called a covariant functor or simply functor, since we shall not deal with contravariant ones.

An element $X$ of a category $C^{\bullet}$ is called inversible if there exists its inverse $\sigma X \in C$ such that $\sigma X . X=a X$ and $X . \sigma X=b X$. This inverse is then uniquely determined by $X$ and $a \sigma X=b X, b \sigma X=a X$. The category $C^{\bullet}$ is called a groupoid if $\sigma$ is a oneto-one mapping of the class $C$ onto itself. The groupoid $C^{*}$ is called transitive if there exists for each pair $\left(e, e^{\prime}\right)$ of unities a morphism $X: e \rightarrow e^{\prime}$. Note that a groupoid is a group iff all the unities coincide.

Definition 1.2. ([6]) A category of operators on a class $E$ is a category $C^{\bullet}$ with a composition $(\theta, z) \rightarrow \theta . z$ which is defined for some pairs $(\theta, z), \theta \in C, z \in E$, $\theta . z \in E$ and satisfies

1. If either $\theta .\left(\theta^{\prime} . z\right)$ or $\left(\theta . \theta^{\prime}\right) . z$ are defined then both they are defined and equal.

2. If $\theta . \theta^{\prime}$ and $\theta^{\prime} . z$ are defined then also $\theta .\left(\theta^{\prime} . z\right)$ is defined.

3. If $e$ is a unity of $C^{\bullet}$ and $e . z$ is defined then $e . z=z$.

4. Each $\theta \in C$ admits at least one $z \in E$ such that $\theta . z$ is defined and each $z \in E$ admits at least one $\theta \in C$ such that $\theta . z$ is defined.

These axioms imply the existence of a projection $p$ of $E$ onto the subclass $B \subset C$ of unities in the category $C^{*}$ such that $\theta . z$ is defined iff $p(z)=a \theta$. In this case we have also $p(\theta . z)=b \theta$.

Let $C^{\bullet}$ be a category of operators on the class $E$. We denote by $C_{E}^{*}$ the category (c.f. [6]), consisting of pairs $(\theta, z), \theta \in C, z \in E$ such that $\theta . z$ is defined, with the composition

$$
\left(\theta^{\prime}, z^{\prime}\right) \cdot(\theta, z)=\left(\theta^{\prime} \cdot \theta, z\right)
$$

defined iff $z^{\prime}=\theta . z$. The unities are the pairs $(p(z), z)$ which we identify with the elements $z$ of $E$. Hence we have for the natural projections $a_{E}(\theta, z)=z$ and $b_{E}(\theta, z)=$ $=\theta . z$. If $\theta$ is inversible then also $(\theta, z)$ is inversible, its inverse being $(\sigma \theta, \theta . z)$. 
Thus if $C^{\bullet}$ is a groupoid then also $C_{E}^{*}$ is a groupoid. There is also a natural functor $(\theta, z) \rightarrow \theta$ from the category $C_{E}^{\bullet}$ onto $C^{\bullet}$.

In the next we shall deal only with the case when $C^{*}$ is a groupoid and write $\Phi$ instead of $C$. We have then a groupoid of operators on $E$ and the structure defined on $E$ in this way is called the fibred structure associated with the groupoid $\Phi^{\bullet}$ (c.f. [4]). For the sake of simplicity we restrict ourselves only to the case when the underlying class $\Phi$ of the groupoid $\Phi^{\bullet}$ as well as the class $E$ are sets. Especially a transitive groupoid is now a groupoid in the sense of Brandt.

If $\Phi^{\bullet}$ is a groupid of operators on $E, B \subset E$ being the subset of its unities, we say that $\Phi^{\bullet}$ is an effective groupoid of operators on $E$ if in addition $\{p(z)=a \theta \Rightarrow \theta . z=$ $=z\} \Rightarrow \theta \in B$ holds. In general let us define by

$$
K=\{\theta \in \Phi \mid p(z)=a \theta \Rightarrow \theta \cdot z=z\}
$$

the subgroupoid $K^{\bullet}$ of the groupoid $\Phi^{\bullet}$ operating on $E$. The $\operatorname{cosets} \theta . K=K . \theta$ form a groupoid $\Phi^{\bullet} / K^{\bullet}=(\Phi / K)^{\bullet}$ which is an effective groupoid of operators on $E$. The relation $\theta \cdot K=K . \theta$ follows from $\theta \cdot \theta^{\prime}=\left(\theta \cdot \theta^{\prime} . \sigma \theta\right) . \theta$ and $\theta^{\prime} \in K \Rightarrow \theta . \theta^{\prime}$. . $\sigma \theta \in K$. A subgroupoid of the groupoid $\Phi^{\bullet}$ is called stable if all the elements $\theta$ of the subgroupoid satisfy $a \theta=b \theta$. Especially the subgroupoid $K^{\bullet}$ is stable. Note that $\Phi^{\bullet}$ operates effectively on $E$ iff $\Phi_{E}^{\cdot}$ has the following property: If the inverse image of $\theta \in \Phi$ under the functor $(\theta, z) \rightarrow \theta$ is a stable subgroupoid of $\Phi_{E}^{\cdot}$ then $\theta \in B$. On the other hand the subgroupoid $K^{\bullet} \subset \Phi^{\bullet}$ defined in (1.1) consists of all the elements $\theta \in \Phi$ for which this inverse image is stable. We say also that $\Phi_{E}^{\cdot}$ is simple or that $\Phi$. operates simply on $E$ if each stable subgroupoid of $\Phi_{E}^{\cdot}$ is mapped into $B$ by the natural functor $(\theta . z) \rightarrow \theta$. We say that $\Phi^{\cdot}$ operates transitively on $E$ if $\Phi_{E}^{\cdot}$ is transitive.

Differentiable manifolds, mappings and differentiable structures defined below are always assumed to be of class $C^{\infty}$. We restrict ourselves to manifolds which are at the same time real, connected and Hausdorff spaces of finite dimension.

Definition 1.3. ([4], [7]) We say that the groupoid $\Phi^{\bullet}$ is differentiable if the set $\Phi$ has the structure of a differentiable manifold, and the mappings $a, b$, and $\left(\theta, \theta^{\prime}\right) \rightarrow$ $\rightarrow \theta . \theta^{\prime}$ are differentiable mappings, $a, b$ being projections of the maximal rank (i.e. $a^{*}$ and $b^{*}$ map the tangent space $T(\Phi)_{\theta}$ onto $T(B)_{a \theta}$ and $T(B)_{b \theta}$ respectively for each $\theta \in \Phi, B \subset \Phi$ denoting as always the subset of unities). Thus we suppose that $B \subset \Phi$ is a proper submanifold (i.e. with the induced topology, c.f. [7]) and $\left(\theta, \theta^{\prime}\right) \rightarrow$ $\rightarrow \theta . \theta^{\prime}$ is defined on a proper submanifold $M \subset \Phi \times \Phi$ defined implicitly by the relation $\left(\theta, \theta^{\prime}\right) \in M \Leftrightarrow a \theta=b \theta^{\prime}$.

The mapping $\sigma$ is then necessarily differentiable (c.f. [7], theorem 1) and it is not difficult to see that $B$ is also regularly imbedded into $\Phi$. This means, denoting by $\sim: B \rightarrow \Phi$ this imbedding, that $\sim *$ injects $T(B)_{x}$ into $T(\Phi)_{\tilde{x}}$ for each $x \in B$. The same holds about the submanifold $M$ of composable elements (c.f. below).

A differentiable groupoid $\Phi^{\bullet}$ is called locally trivial if there exists a fixed point $c \in B$ and a covering of $B$ by open sets $U$ such that for each $U$ there is a differentiable 
mapping $\theta_{U}: U \rightarrow \Phi$ satisfying $a \theta_{U}=c$ and $b \theta_{U}=\mathrm{id}_{U}$. We write also $\theta_{U}(u)=\theta_{u}$. A locally trivial differentiable groupoid is necessarily transitive. Note that this definition of local triviality differs formally from that of Ehresmann (c.f. [4] or [7]), however, since $B$ is here supposed connected, both they are equivalent.

Definition 1.4. ([4], [7]) Let $\Phi^{\bullet}$ be a groupoid of operators on a manifold $E . \Phi^{\bullet}$ is then called a differentiable groupoid of operators on $E$ if $\Phi^{\cdot}$ is a differentiable groupoid and

1. $p: E \rightarrow B$ is a differentiable mapping of maximal rank.

2. The composition $(\theta, z) \rightarrow \theta . z$ being defined on a proper submanifold of $\Phi \times E$ is differentiable.

It could be shown that if $\Phi^{*}$ is a differentiable groupoid of operators on the manifold $E$ then $\Phi_{E}^{\cdot}$ is a differentiable groupoid.

Let $E=E\left(B, F, G^{*}, H\right)$ be a differentiable fibre bundle in the usual meaning of the word with the basis $B$, fibre type $F$, structure group $G^{*}$ and associated principal fibre bundle $H$. In general, however, we do not suppose that $G^{*}$ operates on $F$ effectively. The associated groupoid $\Phi^{\bullet}=H H^{-1}$ consists of all admissible isomorphisms $\theta=$ $=h^{\prime} h^{-1}: E_{x} \rightarrow E_{y}, h \in H_{x}, h^{\prime} \in H_{y}$ of one fibre in $E$ onto another one in the same bundle. If $\theta: E_{x} \rightarrow E_{y}$ is an element of $\Phi^{*}$ then $a \theta$ is the identity of $E_{x}$ and $b \theta$ the identity of $E_{y}$. Identifying these identities with the elements $x \in B$ or $y \in B$ respectively we have $a \theta=x=p(h), b \theta=y=p\left(h^{\prime}\right)$ and the basis $B$ represents the submanifold of unities in $\Phi^{*}$. It is now not dificult to see that $\Phi^{*}$ is a differentiable groupoid of operators on $E$ and the associated fibred structure on $E$ is induced by the underlying fibre bundle structure. Moreover $\Phi^{*}$ is locally trivial and it operates effectively on $E$ iff $G$ • operates effectively on $F$. Note that $\Phi$ itself can be given a fibre bundle structure in two manners (c.f. [3]):

A) $\Phi$ is a differentiable fibre bundle with the basis $B \times B$, projection $a \times b$, fibre type $G$ and structure group $(G \times G)^{\cdot}$ operating on the left on $G$ by $s \rightarrow u . s . v^{-1}$, $u, s, v$ belonging to $G$. $\Phi$ can be also identified with the equivalence classes of $H \times H$, the equivalence being given by $\left(h, h^{\prime}\right) \sim\left(h s, h^{\prime} s\right)$.

B) $\Phi$ is a differentiable fibre bundle with the basis $B$, projection $a$, fibre type $H$ and structure group $G^{*}$ contragradient to $G^{*}$. The associated principal fibre bundle is isomorphic with $H$, the correspondence between elements $h \in H$ and isomorphisms $\hat{h}: H \rightarrow H H^{-1}$ being given by $h \rightarrow\left(h^{\prime} \rightarrow h\left(h^{\prime}\right)^{-1}\right)$.

Conversely suppose that $\Phi^{\bullet}$ is any locally trivial differentiable groupoid, $K^{\bullet}$ any stable subgroupoid of $\Phi^{*}$ (i.e. such that its elements $\theta$ satisfy $a \theta=b \theta$ ). Then $H=H\left(\Phi^{*}, K^{*}\right) \subset \Phi / K$ which consists of $\operatorname{cosets} \theta . K$ for which $a \theta=c$ is a principal fibre bundle with the basis $B$ the structure group being $G\left(\Phi^{*}, K^{*}\right) \subset \Phi^{*} \mid K^{*}$ consisting of cosets $\theta . K$ for which $a \theta=b \theta=c$. Here $c \in B$ is an arbitrary but in the next always fixed point given by the local triviality of $\Phi^{\circ}$. These results hold especially 
for $K^{\bullet}=B$. If now moreover $\Phi^{\bullet}$ is a differentiable groupoid of operators on the manifold $E$ then $E$ is a fibre bundle its fibre type being $p^{-1}(c)$ with effective structure group $G\left(\Phi^{\circ}, K^{*}\right)$ and the associated principal fibre bundle $H\left(\Phi^{*}, K^{\circ}\right)$, where $K$ is given as in (1.1). In fact if $U$ is an open subset of $B$ on which $\Phi^{\cdot}$ is trivial then the correspondence $(u, z) \rightarrow \theta_{u} . z$ defines the diffeomorphism of $U \times p^{-1}(c)$ onto $p^{-1}(U)$. Its inverse is $z \in p^{-1}(U) \rightarrow\left(u=p(z), \theta_{u}^{-1} \cdot z\right) \in U \times p^{-1}(c)$. This (effective) fibre bundle structure induces the fibred structure associated with $\Phi^{\bullet}$ (or $\left.(\Phi / K)^{\circ}\right)$ and it is determined uniquely up to an isomorphism depending on the choice of $c \in B$. More generally $H\left(\Phi^{\circ}, B\right)$ ia a (non-effective) principal fibre bundle with the structure group $G\left(\Phi^{*}, B\right)$ operating not necessarily effectively on the fibre type $p^{-1}(c)$ of $E$. We have namely $H\left(\Phi^{\bullet}, K^{*}\right)=H\left(\Phi^{\bullet}, B\right) / K^{\bullet} \subset \Phi^{\bullet} / K^{\bullet}$ and analogously $G\left(\Phi^{*}, K^{*}\right)=$ $=G\left(\Phi^{\bullet}, B\right) / K^{\bullet}$. Denote by $e=\tilde{c}$ in the next the unity of the group $G\left(\Phi^{\circ}, B\right)$. Thus we have proved the

Lemma 1.1. If $\Phi^{\bullet}$ is a (effective) differentiable groupoid of operators on the manifold $E$ then the associated fibred structure on $E$ is induced by a (effective) fibre bundle structure on $E$ if and only if $\Phi^{\bullet}$ is locally trivial. The corresponding fibre bundle structure on $E$ is uniquely determined by $\Phi^{\bullet}$ up to an isomorphism and it is a principal fibre bundle structure iff $\Phi^{\bullet}$ operates transitively and simply on $E$.

It is again not difficult to see that if $\Phi^{\bullet}$ is a differentiable groupoid of operators on the manifold $E$ then the groupoid $\Phi_{E}^{\cdot}$ is locally trivial iff $\Phi^{\bullet}$ is locally trivial and there exists a point $z_{0} \in p^{-1}(c) \subset E$ and a differentiable mapping $g: p^{-1}(c) \rightarrow G=$ $=G\left(\Phi^{\bullet}, B\right)$ such that $g(z) \cdot z_{0}=z$. Especially the local triviality of $\Phi_{E}^{\cdot}$ implies the transitivity of the operation of $\Phi^{\bullet}$ on $E$.

As usual, if $V_{n}$ and $V_{p}$ are two differentiable manifolds we denote by $\tilde{J}^{r}\left(V_{n}, V_{p}\right)$ or $\bar{J}^{r}\left(V_{n}, V_{p}\right)$ or $J^{r}\left(V_{n}, V_{p}\right)$ respectively the manifold of all non-holonomic or semiholonomic or holonomic respectively $r$-jets of local differentiable mappings from $V_{n}$ into $V_{p}([4]) . \alpha$ and $\beta$ denote the source and the target mappings respectively. Further $\tilde{J}_{s}^{r}\left(V_{n}, V_{p}\right)=\left\{X \in \tilde{J}^{r}\left(V_{n}, V_{p}\right) \mid \alpha(X)=s\right\}$ for any $s \in V_{n}$ and analogously in the semiholonomic and holonomic cases. If the manifold $V_{n}$ consists of only one point $c$, i.e. $\operatorname{dim} V_{n}=0$ we have by definition for each other manifold $V_{p}$ and each $x \in V_{p}$ exactly one element of $\tilde{J}^{r}\left(V_{n}, V_{p}\right)$ with target $x$ whatever be the integer $r>0$. In this way one extends the formalism of the jet calcul to manifolds with non-negative dimension. If $V_{n}$ is again an arbitrary differentiable manifold we denote by $\tilde{\Pi}^{r}\left(V_{n}\right) \subset$ $\subset \tilde{J}^{r}\left(V_{n}, V_{n}\right)$ the differentiable groupoid of all inversible non-holonomic $r$-jets the composition in it being the usual composition of jets. Denote further by $\tilde{H}^{r}\left(V_{n}\right)$ the subset of $\tilde{J}^{r}\left(R^{n}, V_{n}\right)\left(n=\operatorname{dim} V_{n}\right)$ consisting of all inversible non-holonomic $r$-jets with source $0 \in R^{n} . \tilde{H}^{r}\left(V_{n}\right)$ is a principal fibre bundle over $V_{n}$ (c.f. [4]) the projection being the target mapping and its elements are called non-holonomic r-frames in $V_{n}$. Similar meanings for $\bar{\Pi}^{r}\left(V_{n}\right), \Pi^{r}\left(V_{n}\right), \bar{H}^{r}\left(V_{n}\right)$ and $H^{r}\left(V_{n}\right)$. The symbol $j_{x}^{r}$ denotes always the $r$-jet of an identity with source and target $x$, the symbol $j_{x[}^{r}[s]$ denotes the $r$-jet of the constant mapping taking a neighbourhood of $x$ into a point $s$, and 
$\hat{J}_{x}^{r}=j_{x}^{r}[x]$. In the notations sometimes to avoid confusion we shall write e.g. $j_{x}^{r}(u \rightarrow$ $\rightarrow f(u)$ ) instead of the usual $j_{x}^{r} f$. From the local expression of the composition of jets we derive the following

Lemma 1.2. Let $V_{m}, V_{n}, V_{p}$ be differentiable manifolds and let $f: V_{n} \rightarrow V_{p}$ be a differentiable injection (projection) of maximal rank, i.e. let $f^{*}: T\left(V_{n}\right) \rightarrow T\left(V_{p}\right)$ be also a differentiable injection (projection). Then $j^{r} f: \tilde{J}^{r}\left(V_{m}, V_{n}\right) \rightarrow \tilde{J}^{r}\left(V_{m}, V_{p}\right)$ is a differentiable injection (projection) of maximal rank. Similarly in the semiholonomic or holonomic cases.

Corollary. If $V_{n}$ is injected and regularly imbedded into $V_{p}$ then $\tilde{J}^{r}\left(V_{m}, V_{n}\right)$ is regularly imbedded into $\tilde{J}^{r}\left(V_{m}, V_{p}\right)$ and we can write then simply $\tilde{J}^{r}\left(V_{m}, V_{n}\right) \subset$ $\subset \tilde{J}^{r}\left(V_{m}, V_{p}\right)$.

Lemma 1.3. Let $V_{p}, B_{1}, B_{2}, B_{2}^{\prime}$ be differentiable manifolds, $B_{1}$ injected and regularly imbedded into $B_{2}$, and $f, f^{\prime}: B_{2} \rightarrow B_{2}^{\prime}$ differentiable mappings such that $x \in B_{1} \Leftrightarrow f(x)=f^{\prime}(x)$. Then $X \in \tilde{J}^{r}\left(V_{p}, B_{1}\right) \subset \tilde{J}^{r}\left(V_{p}, B_{2}\right) \Leftrightarrow\left(j^{r} f\right) X=\left(j^{r} f^{\prime}\right) X$.

Proof. Denoting by $i: B_{1} \rightarrow B_{2}$ the imbedding we have the imbedding $j^{r} i$ : $: \tilde{J}^{r}\left(V_{p}, B_{1}\right) \rightarrow \tilde{J}^{r}\left(V_{p}, B_{2}\right)$ and the relation $f i=f^{\prime} i$ which implies $\left(j^{r} f\right) j^{r} i=\left(j^{r} f^{\prime}\right) j^{r} i$.

Let $(E, B)$ be a pair of differentiable manifolds with a differentiable projection $p_{E}=p: E \rightarrow B$ of maximal rank. Then we call $E$ a fibred manifold over $B$. If now $E$ is such a fibred manifold over $E$, especially if $E=E\left(B, F, G^{*}, H\right)$ is a differentiable fibre bundle, denote by $\widetilde{\mathscr{D}}^{r} E$ or $\overline{\mathscr{D}}^{r} E$ or $\mathscr{D}^{r} E$ its $r$-th non-holonomic or semi-holonomic or holonomic prolongation respectively. This means that e.g. $\tilde{\mathscr{D}}^{r} E$ consists of all non-holonomic $r$-jets of local sections in $E$ over $B$. We have also $\mathscr{D}^{r} E=\{F \in$ $\left.\in \tilde{J}^{r}(B, E) \mid\left(j_{\beta(F)}^{r} p\right) F=j_{\alpha(F)}^{r}\right\}$. We shall study below explicitly the fibre bundle structure of these prolongations (c.f. proposition 1.1).

The prolongation of a composition law in general is now defined as follows. Let $\varphi: M \subset B_{1} \times B_{2} \rightarrow B_{2}$ be a composition given by $\varphi\left(z, z^{\prime}\right)=z \cdot z^{\prime}$, where $B_{1}, B_{2}, M$ are differentiable manifolds, $M$ being injected and regularly imbedded into $B_{1} \times B_{2}$, and $\varphi$ is differentiable. If now $V_{p}$ is another differentiable manifold we define the prolongation of the composition $\varphi$ with respect to $V_{p}$ as follows. Let $Z \in \tilde{J}^{r}\left(V_{p}, B_{1}\right)$, $Z^{\prime} \in \tilde{J}^{r}\left(V_{p}, B_{2}\right)$ such that $\alpha(Z)=\alpha\left(Z^{\prime}\right)$ and $\left(Z, Z^{\prime}\right) \in \tilde{J}^{r}\left(V_{p}, M\right) \subset \tilde{J}^{r}\left(V_{p}, B_{1} \times B_{2}\right)=$ $=\tilde{J}^{r}\left(V_{p}, B_{1}\right) \times \tilde{J}^{r}\left(V_{p}, B_{2}\right)$. We put

$$
Z . Z^{\prime}=\left(j_{\beta(Z), \beta\left(Z^{\prime}\right)}^{r} \varphi\right)\left(Z, Z^{\prime}\right) \in \tilde{J}^{r}\left(V_{p}, B_{2}\right) .
$$

Note that $\alpha\left(Z \cdot Z^{\prime}\right)=\alpha(Z)=\alpha\left(Z^{\prime}\right)$ and $\beta\left(Z \cdot Z^{\prime}\right)=\beta(Z) \cdot \beta\left(Z^{\prime}\right)$. If $V_{n}$ is another differentiable manifold, $X \in \tilde{J}^{r}\left(V_{n}, V_{p}\right), \beta(X)=\alpha(Z)$, we derive easily the relation important for explicite computings

$$
\left(Z \cdot Z^{\prime}\right) X=Z X \cdot Z^{\prime} X .
$$


Here and in the next if the composition is denoted without point or any other symbol (as $(Z, X) \rightarrow Z X$ in (1.3)) it means always the usual composition of jets, i.e. the composition in the "universal" category of all (non-holonomic) $r$-jets (c.f. [8], where this category is denoted by $\left.\left(\tilde{J}^{r, \infty}, A^{r, \infty}\right)\right)$.

We see also that if $Z$ and $Z^{\prime}$ are semi-holonomic or holonomic jets then the same is true about $Z . Z^{\prime}$. E.g. if $G^{\bullet}$ is a Lie group then $\widetilde{T}_{p}^{r}(G)^{\bullet}, \bar{T}_{p}^{r}(G)^{\bullet}$ and $T_{p}^{r}(G)^{\bullet}\left(p^{r}\right.$ velocities on $G$ ) are Lie groups and if $G^{*}$ operates (on the left) on the differentiable manifold $V_{n}$ then $\widetilde{T}_{p}^{r}(G)^{\cdot}$ or $\bar{T}_{p}^{r}(G)^{\cdot}$ or $T_{p}^{r}(G)^{\cdot}$ operate on $\tilde{T}_{p}^{r}\left(V_{n}\right)$ or $\bar{T}_{p}^{r}\left(V_{n}\right)$ or $T_{p}^{r}\left(V_{n}\right)$ respectively.

More generally let $\Phi^{\bullet}$ be a differentiable groupoid. Then $M \subset \Phi \times \Phi$ consists of composable pairs $\left(\theta, \theta^{\prime}\right)$, i.e. such that $a \theta=b \theta^{\prime}$. It is a regularly imbedded submanifold of $\Phi \times \Phi$. In fact, $M$ is the inverse image of the diagonal in $B \times B$ under the projection $a \times b: \Phi \times \Phi \rightarrow B \times B$ which is of maximal rank.

Remark. Note that the restriction of $a \times b$ onto the diagonal in $\Phi \times \Phi$ defines a mapping $(a, b)$ from $\Phi$ onto $B \wedge B \subset B \times B$, where $B \wedge B$ consists of pairs $\left(e, e^{\prime}\right)$ such that there exists an element $\theta \in \Phi, \theta: e \rightarrow e^{\prime}$. In [8] the groupoid $\Phi^{\bullet}$ is called regularly differentiable (however, under more general assumptions concerning the underlying differentiable structure) if this mapping $(a, b)$ is a projection of maximal rank. Note that $\Phi^{\bullet}$ is transitive iff $B \wedge B=B \times B$ and that it is locally trivial iff it is regularly differentiable and $B \wedge B=B \times B$. E.g. the differentiable groupoid $\Phi^{\bullet}$ over a connected $B$ as defined in [17] is necessarily transitive, regularly differentiable and locally trivial.

In the preceding situation if now $V_{p}$ is a differentiable manifold we can define the differentiable groupoid $\tilde{J}^{r}\left(V_{p}, \Phi\right)^{\bullet}$ with the composition law being the prolongation of the composition in $\Phi^{*}$. The unities in $\tilde{J}^{r}\left(V_{p}, \Phi\right)^{\circ}$ are exactly the elements of $\tilde{J}^{r}\left(V_{p}, B\right)$. Let us write simply $a: \tilde{J}^{r}\left(V_{p}, \Phi\right) \rightarrow \tilde{J}^{r}\left(V_{p}, B\right)$ or $b: \tilde{J}^{r}\left(V_{p}, \Phi\right) \rightarrow \tilde{J}^{r}\left(V_{p}, B\right)$ or $\sigma:$ $: \tilde{J}^{r}\left(V_{p}, \Phi\right) \rightarrow \tilde{J}^{r}\left(V_{p}, \Phi\right)$ instead of $j^{r} a$ or $j^{r} b$ or $j^{r} \sigma$ respectively. If now $X \in \tilde{J}^{r}\left(V_{p}, \Phi\right)$ then $a X$ or $b X$ or $\sigma X$ are its right unit or left unit or its inverse respectively, i.e. $a X=\sigma X . X, b X=X . \sigma X$. The composition $X . X^{\prime}$ is defined iff $a X=b X^{\prime}$. This implies $\alpha\left(X^{\prime}\right)=\alpha\left(X^{\prime}, \sigma X^{\prime}\right)=\alpha\left(b X^{\prime}\right)=\alpha(a X)=\alpha(\sigma X \cdot X)=\alpha(X)$. Thus we see according to lemma 1.2 and to its corollary that $\tilde{J}^{r}\left(V_{p}, \Phi\right)^{*}$ is a differentiable groupoid and it is a disjoint union of the groupoids $\tilde{J}_{s}^{r}\left(V_{p}, \Phi\right)^{\bullet}, s \in V_{p}$. If $\Phi^{\bullet}$ is transitive then each of these components is also transitive $\left.{ }^{1}\right)$ and if $\Phi^{\bullet}$ is locally trivial then the same is true about each $J_{s}^{r}\left(V_{p}, \Phi\right)^{\circ}$ (c.f.ch. 3 in the more general case).

Analogously if $\Phi^{*}$ is a differentiable groupoid of operators on the manifold $E$ then the underlying manifold of $\Phi_{E}^{\cdot}$ (i.e. the manifold of composable pairs $(\theta, z), \theta \in \Phi$, $z \in E)$ is regularly imbedded into $\Phi \times E$. Thus $\tilde{J}^{r}\left(V_{p}, \Phi\right)^{\circ}$ becomes a differentiable groupoid of operators on the manifold $\tilde{J}^{r}\left(V_{p}, E\right)$ the operation being given by the

\footnotetext{
${ }^{1}$ ) It can be shown in general that each groupoid is decomposable into a disjcint union of transitive groupoids. Here disjoint means that if two elements of the groupoid are composatle then they necessarily belong to the same component of the decomposition.
} 
prolongation of the operation law of $\Phi^{\bullet}$ on $E$. Note, however, that unless $V_{p}$ consists of only one point, $\tilde{J}^{r}\left(V_{p}, E\right)$ is not a fibre bundle over $\tilde{J}^{r}\left(V_{p}, B\right)$ since $\tilde{J}^{r}\left(V_{p}, \Phi\right)^{\bullet}$ is not locally trivial. In fact, we have seen that the composability of $X, X^{\prime} \in \tilde{J}^{r}\left(V_{p}, \Phi\right)$ implies $\alpha(X)=\alpha\left(X^{\prime}\right)$. Nevertheless we make the following important remark. Since $H=H\left(\Phi^{*}, B\right)$ and $G=G\left(\Phi^{*}, B\right)$ are regularly imbedded into $\Phi$, this being an almost trivial consequence of the very definition of the differentiable structure in $\Phi^{\circ}$ and that of $H \subset \Phi$ and $G \subset \Phi$, we have $\tilde{J}^{r}\left(V_{p}, H\right) \subset \tilde{J}^{r}\left(V_{p}, \Phi\right)$ and $\tilde{J}^{r}\left(V_{p}, G\right) \subset$ $\subset J^{r}\left(V_{p}, \Phi\right)$ and according to lemma 1.3 we have

$$
\begin{aligned}
& X \in \tilde{J}^{r}\left(V_{p}, H\right) \Leftrightarrow a X=j_{\alpha(X)}^{r}[c] \\
& X \in \tilde{J}^{r}\left(V_{p}, G\right) \Leftrightarrow a X=b X=j_{\alpha(X)}^{r}[c] .
\end{aligned}
$$

According to lemma 1.3 again (we take $B_{2}=\Phi \times E, B_{2}^{\prime}=B \times B$ and $B_{1}=\Phi_{E}$ ) one can canonically identify $\tilde{J}^{r}\left(V_{p}, \Phi\right)_{E^{\prime}}^{\cdot}-$ with $E^{\prime}$ equal to $\tilde{J}^{r}\left(V_{p}, E\right)$ - with $\tilde{J}^{r}\left(V_{p}, \Phi_{E}\right)^{\circ}$. Hence if $\Phi^{*}$ operates transitively on $E$ then each $\tilde{J}_{s}^{r}\left(V_{p}, \Phi\right)^{\cdot}$ operates transitively on $\tilde{J}_{s}^{r}\left(V_{p}, E\right), s \in V_{p}$. All these considerations remain valid, of course, also in the semiholonomic and holonomic cases.

If $\Phi^{\bullet}$ is a differentiable groupoid as before denote $\tilde{\Phi}^{r} \subset \tilde{J}^{r}(B, \Phi)$ the subset of all $X$ for which $a X=j_{x}^{r}, x=\alpha(X)$ and $b X \in \widetilde{\Pi}^{r}(B)$. We define the composition $\left(X, X^{\prime}\right) \rightarrow$ $\rightarrow X \bullet X^{\prime}$ by

$$
X \bullet X^{\prime}=\left(X b X^{\prime}\right) \cdot X^{\prime}
$$

for $X, X^{\prime} \in \widetilde{\Phi}^{r}, \alpha(X)=\beta\left(b X^{\prime}\right)$. This defines a differentiable groupoid $\widetilde{\Phi}^{r \bullet}$ called the $r$-th non-holonomic prolongation of $\Phi^{*}$ (c.f. [4], [8]). Since $X=X . a X=X a X$. $. a X=(X b a X) . a X$ we see that the right unit of $X$ is $a X=j_{x}^{r}$ and similarly the left unit of $X$ is $(b X)(b X)^{-1}=j_{x}^{r}$ with $y=\beta(b X)=b \beta(X)$. The inverse of $X$ is $\sigma X(b X)^{-1}$. Thus the unities in $\widetilde{\Phi}^{r \bullet}$ are exactly all the $r$-jets of identities on $B$. Identifying as usually the jet $j_{x}^{r}$ with $x \in B$ we can assume $B$ to be the submanifold of unities in $\tilde{\phi}^{r} \bullet$. Analogously one defines the $r$-th semi-holonomic prolongation $\bar{\phi}^{r} \bullet$ and the $r$-th holonomic prolongation $\Phi^{r \bullet}$ of the differentiable groupoid $\Phi^{\bullet}$. Any of the prolongations of $\Phi^{\bullet}$ is transitive if $\Phi^{\bullet}$ is transitive and we have also the

Lemma 1.4. If the differentiable groupoid $\Phi^{\bullet}$ is locally trivial then each of its prolongations $\widetilde{\Phi}^{r \bullet}$ or $\bar{\Phi}^{r \bullet}$ or $\Phi^{r \bullet}$ is locally trivial.

Proof. We shall give it only in the non-holonomic case. Let $c \in B$ and the open covering $\left\{U_{\alpha}\right\}$ of $B$ be given by the local triviality of $\Phi^{*}$. Let $c \in U_{c}$ and let $U$ be any of the sets $U_{\alpha}$. We may suppose that $U_{c}$ is diffeomorphic with $U$. Define $\Theta_{U}: u \in U \rightarrow$ $\rightarrow \Theta_{u} \in \tilde{\Phi}^{r}$ by $\Theta_{u}=j_{c}^{r} \hat{\theta}_{u}$ where $\hat{\theta}_{u}(x)=\theta_{\varphi(x)} . \theta_{x}^{-1}$, whereby $\theta_{x}$ for $x \in U_{c}$ and $\theta_{y}$ for $y=\varphi(x) \in U$ are given by the local triviality of $\Phi^{*}$ and $\varphi: U_{c} \rightarrow U$ is a diffeomorphism for which $\varphi(c)=u$. We have $a \Theta_{u}=j_{c}^{r}\left(a \hat{\theta}_{u}\right)=j_{c}^{r}\left(x \rightarrow \theta_{x}^{-1}\right)=j_{c}^{r} ; \quad b \Theta_{u}=$ $=j_{c}^{r}\left(b \hat{\theta}_{u}\right)=j_{c}^{r}\left(b \theta_{\varphi}\right)=j_{c}^{r} \varphi \in \tilde{\Pi}^{r}(B)$ and thus $\Theta_{u} \in \widetilde{\Phi}^{r}$ (even $\left.\Theta_{u} \in \Phi^{r}\right)$ and $u \rightarrow \Theta_{u}$ is differentiable if we chose suitably the dependence of $\varphi$ upon $u$. Since moreover $\left(b \Theta_{u}\right)\left(b \Theta_{u}\right)^{-1}=j_{y}^{r}$ with $y=b \hat{\theta}_{u}(c)=b \theta_{\varphi(c)}=\varphi(c)=u$ the lemma is proved. 
Let $\Phi^{\bullet}$ be a locally trivial differentiable groupoid. It can be considered as a fibre bundle with the basis $B \times B$ (c.f. the alternative A) above) and hence $G\left(\tilde{\Phi}^{r \bullet}, B\right)$ can be identified with the group $G\left(\widetilde{\Phi}_{0}^{r \bullet}, R^{n}\right)$, where $n=\operatorname{dim} B$ and $\widetilde{\Phi}_{0}^{\cdot}$ is the trivial groupoid $R^{n} \times R^{n} \times G$ with $G=G\left(\Phi^{*}, B\right)$ and the composition $(x, y, \theta) .\left(x^{\prime}, y^{\prime}, \theta^{\prime}\right)=$ $=\left(x^{\prime}, y, \theta \cdot \theta^{\prime}\right)$ defined iff $y^{\prime}=x$. Thus $a(x, y, \theta)=x, b(x, y, \theta)=y$ in $\Phi_{0}^{\circ}$. We have on the other hand the identification $\tilde{J}^{r}\left(R^{n}, \Phi_{0}\right)=R^{n} \times \tilde{J}_{0}^{r}\left(R^{n}, \Phi_{0}\right)=R^{n} \times$ $\times \tilde{J}_{0}^{r}\left(R^{n}, R^{n}\right) \times \tilde{J}_{0}^{r}\left(R^{n}, R^{n}\right) \times \tilde{T}_{n}^{r}(G)$, where $X \in \tilde{J}^{r}\left(R^{n}, \Phi_{0}\right)$ is identified with the quadruple $\left(\alpha(X), a X, b X, X_{0}\right), X_{0} \in \widetilde{T}_{n}^{r}(G)$. If the $X$ belongs moreover to $\widetilde{\Phi}_{0}^{r}$ then it can be characterized by the triple $\left(\alpha(X), b X, X_{0}\right) \in R^{n} \times \tilde{J}^{r}\left(R^{n}, R^{n}\right) \times \widetilde{T}_{n}^{r}(G)$, where $b X$ is invertible and if one choses $c=0$ in $R^{n}$ one can characterize the element $X \in$ $\in G\left(\tilde{\Phi}_{0}^{r \bullet}, R^{n}\right)$ by the pair $\left(b X, X_{0}\right) \in \tilde{L}_{n}^{r} \times \tilde{T}_{n}^{r}(G)$, where $\tilde{L}_{n}^{r}=\left\{y \in \tilde{\Pi}^{r}\left(R^{n}\right) \mid \alpha(y)=\right.$ $=\beta(y)=0\}$. The composition $X \bullet X^{\prime}$ is at the same time taken into the pair $(b(X \bullet$ - $\left.\left.X^{\prime}\right), X_{0} \bullet X_{0}^{\prime}\right)=\left(b X b X^{\prime}, X_{0} b X_{0}^{\prime} \cdot X_{0}^{\prime}\right)$. The composition in the group $G\left(\tilde{\Phi}_{0}^{r \bullet}, R^{n}\right)=$ $=\left(\tilde{L}_{n}^{r} \times \tilde{T}_{n}^{r}(G)\right)^{\bullet}$ is thus given by (c.f. [4])

$$
(y, S) \bullet\left(y^{\prime}, S^{\prime}\right)=\left(y y^{\prime}, S y^{\prime} . S^{\prime}\right) .
$$

In order to get explicite form of this representation $G\left(\tilde{\Phi}^{r}, B\right) \rightarrow\left(\tilde{L}_{n}^{r} \times \tilde{T}_{n}^{r}(G)\right)^{\bullet}$ suppose that $c \in B$ and the open subset $U_{c}$ of $B, c \in U_{c}$ together with $u \rightarrow \theta_{u}, u \in U_{c}$ are given by the local triviality of $\Phi^{*}$ and denote by $\varphi$ the $r$-jet of a diffeomorphism of $U_{c}$ into $R^{n}$ with source $c$ and target 0 and $\Sigma=j_{c}^{r}\left(u \rightarrow \theta_{u}\right)$. We may suppose always that $c \in B, \varphi$ and $\Sigma$ connected with the locally trivial differentiable groupoid $\Phi^{\bullet}$ are fixed. It is now not difficult to derive from the above considerations that if $Y \in$ $\in G\left(\tilde{\Phi}^{r \bullet}, B\right) \subset \tilde{\Phi}^{r \bullet}$ then the corresponding pair $(y, S) \in \tilde{L}_{n}^{r} \times \tilde{T}_{n}^{r}(G)$ is given by

$$
\left.y=\varphi b Y \varphi^{-1} ; \quad S=\{\sigma \Sigma b Y . Y . \Sigma)\right\} \varphi^{-1} .
$$

The associated principal fibre bundle $H\left(\tilde{\Phi}^{r \bullet}, B\right)$ can be identified analogously with the manifold of all elements in $\tilde{H}^{r}(B) \times \tilde{\mathscr{D}}^{r} H$ which are projected onto the diagonal in $B \times B$. We denote this manifold by $\tilde{H}^{r}(B) \bigotimes \tilde{\mathscr{D}}^{r} H$. In fact, considering now the locally trivial groupoid $\Phi^{\bullet}$ as a fibre bundle over $B$ (the alternative $B$ ) above) we have $\widetilde{\Phi}^{r}=\left\{X \in \widetilde{\mathscr{D}}^{r} \Phi \mid b X \in \widetilde{\Pi}^{r}(B)\right.$ and $H\left(\tilde{\Phi}^{r \bullet}, B\right)=\left\{X \in \tilde{\Phi}^{r} \mid \alpha(X)=c\right\}$. We see that $H\left(\tilde{\Phi}^{r \bullet}, B\right)$ can be identified with the set of elements $X \in \tilde{J}_{0}^{r}\left(R^{n}, H\right)$ for which $p_{H} X \in$ $\in \tilde{H}^{r}(B)$, i.e. with the set of pairs $X_{1} \in \tilde{H}^{r}(B), X_{2} \in \tilde{\mathscr{D}}^{r} H$ for which $\alpha\left(X_{2}\right)=\beta\left(X_{1}\right)$, where $H=H\left(\Phi^{\bullet}, B\right)$. Explicitly if $X \in H\left(\widetilde{\Phi}^{r \bullet}, B\right)$ and $\varphi, \Sigma$ are given as above in (1.6) we get for the corresponding $X_{1} \in \tilde{H}^{r}(B), X_{2} \in \tilde{D}^{r} H$ the formula

$$
X_{1}=b X \varphi^{-1} ; \quad X_{2}=(X . \Sigma)(b X)^{-1} .
$$

Hence our identifications imply immediately that $\tilde{H}^{r}(B)$ 冈 $\tilde{D}^{r} H$ is a principal fibre bundle (associated de facto with the groupoid $\tilde{\Phi}^{r \bullet}$ ) with the structure group $\left(\tilde{L}_{n}^{r} \times\right.$ $\left.\times \tilde{T}_{n}^{r}(G)\right)^{\bullet}$. Explicitly, the pair $(y, S)$ operates (on the right) on the pair $\left(X_{1}, X_{2}\right)$ as

$$
\left(X_{1}, X_{2}\right) \bullet(y, S)=\left(X_{1} y, X_{2} . S\left(X_{1} y\right)^{-1}\right) .
$$


In fact, from (1.7) for the composition, using (1.6) and (1.7) for the components, we get $b(X \bullet Y) \varphi^{-1}=b X b Y \varphi^{-1}=X_{1} y$ and $(X \bullet Y . \Sigma) b(X \bullet Y)^{-1}=(X b Y . Y . \Sigma)$. $\cdot(b Y)^{-1}(b X)^{-1}=X(b X)^{-1} \cdot \Sigma(b X)^{-1} \cdot \sigma \Sigma(b X)^{-1} \cdot Y(b Y)^{-1}(b X)^{-1} \cdot \Sigma(b Y)^{-1}$. $\cdot(b X)^{-1}=(X . \Sigma)(b X)^{-1} \cdot\{\sigma \Sigma . b Y . Y . \Sigma\}(b Y)^{-1} \varphi^{-1} \varphi(b X)^{-1}=X_{2} \cdot S\left(X_{1} y\right)^{-1}$.

If $\Phi^{\bullet}$ is again a differentiable groupoid (not necessarily locally trivial) operating on the manifold $E$ and $X \in \tilde{\Phi}^{r}, F \in \widetilde{\mathscr{D}}^{r} E_{\alpha(X)}$ define

$$
X \text { ๑ } F=(X . F)(b X)^{-1} .
$$

We have $X \odot\left(X^{\prime} \odot F\right)=\left(X .\left(X^{\prime} . F\right)\left(b X^{\prime}\right)^{-1}\right)(b X)^{-1}=\left(X b X^{\prime} . X^{\prime} . F\right)\left(b X^{\prime}\right)^{-1}$. $.(b X)^{-1}=\left(X \bullet X^{\prime}\right) \bullet F$ and it is not difficult to see that $\tilde{\Phi}^{r}$ is a differentiable groupoid of operators on $\widetilde{\mathscr{D}}^{r} E$ the associated projection $p: \widetilde{\mathscr{D}}^{r} E \rightarrow B$ being the source mapping. If $\Phi^{\bullet}$ is locally trivial, then $E$ is a fibre bundle $E\left(B, F, G^{\bullet}, H\right)$ and according to lemma 1.4 also $\tilde{\mathscr{D}}^{r} E$ is a fibre bundle. Its fibre type $p^{-1}(c)$ can be identified with $\widetilde{T}_{n}^{r}(F)$, whereas $\left(\tilde{L}_{n}^{r} \times \widetilde{T}_{n}^{r}(G)\right)^{\bullet}$ operates on $\tilde{T}_{n}^{r}(F)$ on the left as $(y, S) \in \tilde{L}_{n}^{r} \times \widetilde{T}_{n}^{r}(G)$, $f \in \widetilde{T}_{n}^{r}(F) \Rightarrow$

$$
(y, S) \odot f=(S . f) y^{-1} .
$$

Analogous results hold in the semi-holonomic and holonomic cases. Thus we have the

Proposition 1.1. If $\Phi^{\bullet}$ is a differentiable groupoid of operators on the manifold $E$ then $\widetilde{\Phi}^{r \bullet}$ or $\bar{\Phi}^{r}$ or $\Phi^{r \bullet}$ is a differentiable groupoid of operators on $\tilde{\mathscr{D}}^{r} E$ or $\overline{\mathscr{D}}^{r} E$ or $\mathscr{D}^{r} E$ respectively. If $\Phi^{\bullet}$ is locally trivial then $\tilde{\mathscr{D}}^{r} E$ is a fibre bundle over $B$ with the fibre type $\widetilde{T}_{n}^{r}(F)$, associated principal fibre bundle $\tilde{H}^{r}(B) \otimes \tilde{D}^{r} H$ and structure group $\left(\tilde{L}_{n}^{r} \times \widetilde{T}_{n}^{r}(G)\right)^{\bullet}$. The composition in $\left(\tilde{L}_{n}^{r} \times \tilde{T}_{n}^{r}(G)\right)^{\bullet}$ is given by (1.5), the operation of $\left(\widetilde{L}_{n}^{r} \times \widetilde{T}_{n}^{r}(G)\right)^{\bullet}$ on $\tilde{H}^{r}(B) \bigotimes \tilde{\mathscr{D}}^{r} H$ or $\widetilde{T}_{n}^{r}(F)$ is given by (1.8) or (1.10) respectively. Analogously in the semi-holonomic and holonomic cases.

Remark. We call an explicite chart of the fibre bundle $E(B, F, G \cdot H)$ a triple $\left(U, h_{x}, \varphi\right)$, where $(U, \varphi)$ is a chart of the basis $B$ and $x \rightarrow h_{x}$ is a differentiable section over $U$ in $H, h_{x}: F \rightarrow E_{x}$ (c.f. [18]). If there is given such an explicite chart then the local section $\sigma: U \rightarrow E$ can be identified with a differentiable mapping $\sigma_{0}: U \rightarrow F$ and for a fixed $q \in U$ the $r$-jet $j_{q}^{r} \sigma$ can be identified with the system of "coordinates" $i(\sigma)=\left(\sigma_{0}(q),\left(\partial_{i} \sigma_{0}\right)(q)\right) \in T_{n}^{r}(F)$. If $\left(U, h_{x}^{\prime}, \varphi^{\prime}\right)$ is another explicite chart we get the differentiable mapping $g \sigma_{0}: U \rightarrow F$, where $g: U \rightarrow G$ and $j_{q}^{r} \sigma$ is now to be identified with the system of coordinates $i^{\prime}(\sigma)=\left(\left(g \sigma_{0}\right)(q),\left(\partial_{i^{\prime}}\left(g \sigma_{0}\right)\right)(q)\right)$. We may assume $\varphi(q)=\varphi^{\prime}(q)=0$ and denoting by $y$ the $r$-jet at 0 of the change of the coordinates $\varphi^{\prime} \varphi^{-1}: x^{i} \rightarrow x^{i^{\prime}}$ and $S=j_{0}^{r}\left(g \varphi^{-1}\right)$ we derive easily the relation $i^{\prime}(\sigma)=(y, S) i(\sigma)$, where the composition is defined by (1.10). Thus we see that the fibred structure in $\mathscr{D}^{r} E$ with the structure group $\left(L_{n}^{r} \times T_{n}^{r}(G)\right)^{\bullet}$ defined by means of the operation of $\Phi^{r \bullet}$ on $\mathscr{D}^{r} E$ in (1.9) (where $\Phi^{*}$ is now, of course, the associated groupoid of $E\left(B, F, G^{*}, H\right)$ ) is quite natural. It expresses the fact that the local coordinates of an $r$-jet should be the derivatives of the local coordinates of the section with respect to the local coordi- 
nates in the basis. Conversely, it could be shown that this request determines the composition law in $\left(L_{n}^{r} \times T_{n}^{r}(G)\right)^{\bullet}$ as given by $(1.5)$ and the definition of the $r$-th holonomic prolongation of the associated groupoid.

Let $\Phi^{\bullet}$ be again in general a differentiable groupoid of operators on the manifold $E$. Applying the prolongation procedure to the differentiable groupoid $\Phi_{E}^{*}$ we obtain the differentiable groupoid $\tilde{\Phi}_{E}^{r \bullet}$ defined as follows. $\widetilde{\Phi}_{E}^{r \bullet}$ consists of all elements $X \in$ $\in \tilde{J}^{r}(E, \Phi)$ for which $a X=j_{\alpha(X)}^{r} p$ and $X \cdot j_{\alpha(X)}^{r} \in \tilde{\Pi}^{r}(E)$. Note that the first condition implies for each $X \in \tilde{\Phi}_{E}^{r}$ the property $a \beta(X)=\beta(a X)=p \alpha(X)$, i.e. $\beta(X) \in \Phi$ is composable with $\alpha(X) \in E$. The submanifold of unities in $\widetilde{\Phi}_{E}^{r \bullet}$ is identified with $E$ and the composition is given by

$$
X \bullet X^{\prime}=X\left(X^{\prime} \cdot j_{\alpha\left(X^{\prime}\right)}^{r}\right) \cdot X^{\prime}
$$

defined iff $\beta\left(X^{\prime}\right) . \alpha\left(X^{\prime}\right)=\alpha(X)$. The right and left units of the element $X \in \widetilde{\Phi}_{E}^{r}$ are $\alpha(X)$ and $\beta(X) . \alpha(X)$ respectively. One can see that $\tilde{\Phi}_{E}^{r}$ is really a submanifold of $\tilde{J}^{r}(E, \Phi)$ such that $\alpha$ and $\beta . \alpha$ are projections form $\tilde{\Phi}_{E}^{r}$ onto $E$ of maximal rank and hence $\widetilde{\Phi}_{E}^{r \bullet}$ is a differentiable groupoid.

We finish this chapter with the following remark concerning the effectivness, transitivity and simplicity of the operation of the prolongations. If $\Phi^{\bullet}$ is a differentiable groupoid of operators on the manifold $E, V_{p}$ another differentiable manifold and $K^{\bullet}=K(E)^{\bullet}$ the stable subgroupoid defined in (1.1), denote by $K\left(\tilde{J}^{r}\left(V_{p}, E\right)\right)^{\bullet}$ and $K\left(\tilde{D}^{r} E\right)^{\bullet}$ the kernel of the operation of $\tilde{J}^{r}\left(V_{p}, \Phi\right)^{\bullet}$ on $\tilde{J}^{r}\left(V_{p}, E\right)$ and $\tilde{\Phi}^{r \bullet}$ on $\tilde{\mathscr{D}}^{r} E$ respectively defined analogously as that in (1.1). Now one shows easily by induction on $r$ that the elements $X$ of the stable subgroupoid $\tilde{J}^{r}\left(V_{p}, K\right)^{\bullet}$ of $\tilde{J}^{r}\left(V_{p}, \Phi\right)^{\bullet}$ satisfy $\left(j^{r} p\right) Z=a X, Z \in \tilde{J}^{r}\left(V_{p}, E\right) \Rightarrow X . Z=Z$, i.e. $\tilde{J}^{r}\left(V_{p}, K\right) \subset K\left(\tilde{J}^{r}\left(V_{p}, E\right)\right)$. Analogously $\tilde{K}^{r} \subset K\left(\tilde{D}^{r} E\right)$. We have, however, in general no converse inclusions $\left.{ }^{2}\right)$ and consequently we cannot conclude from the effectivness of the operation of $\Phi^{\bullet}$ to the effectivness of the operation of $\tilde{J}^{r}\left(V_{p}, \Phi\right)^{\bullet}$ or $\tilde{\Phi}^{r \bullet}$. On the other hand one finds easily again by induction on $r$ the canonical isomorphism which gives rise to the identification

$$
\tilde{J}^{r}\left(V_{p}, \Phi / K\right)^{\bullet}=\tilde{J}^{r}\left(V_{p}, \Phi\right)^{\bullet} / \tilde{J}^{r}\left(V_{p}, K\right)^{\bullet} .
$$

An application of lemma 1.3 yields the following result. If $\Phi^{\bullet}$ operates simply on $E$ then $\tilde{J}^{r}\left(V_{p}, \Phi\right)^{\bullet}$ operates simply on $\tilde{J}^{r}\left(V_{p}, E\right)$. Especially if $\Phi^{\bullet}$ is locally trivial and operates simply and transitively on $E$ (i.e. $E$ is a principal fibre bundle) then also each $\tilde{J}_{s}^{r}\left(V_{p}, E\right)$ is a principal fibre bundle. But $\tilde{\mathscr{D}}^{r} E$ is not a principal fibre bundle since its structure group is $\left(\tilde{L}_{n}^{r} \times \tilde{T}_{n}^{r}(G)\right)$ and its fibre type is $\tilde{T}_{n}^{r}(F)=\tilde{T}_{n}^{r}(G)$. In fact, the local triviality (c.f. lemma 1.4) and the transitivity of $\Phi^{\bullet}$ are preserved also by its prolongation $\tilde{\Phi}^{r}$ but not so the simplicity as one can easily see from the explicite formula (1.9).

Analogous remarks relate to the semi-holonomic and holonomic cases.

\footnotetext{
${ }^{2}$ ) As a matter of fact, it remains a problem to prove this actually, i.e. to find an example where the two spaces, in the first or in the second case, are essentially different.
} 


\section{HIGHER ORDER CONNECTIONS IN THE SENSE OF CH. EHRESMANN}

Let $\Phi^{\bullet}$ be a differentiable groupoid of operators on the manifold $E$. Denote as usually $E_{x}=p^{-1}(x) \subset E$ for any $x \in B$.

Definition 2.1. ([5]) A non-holonomic infinitesimal displacement of order $r$ of the fibre $E_{x}$ is any element $\zeta \in \tilde{J}_{0}^{r}\left(R^{n}, \Phi\right), n=\operatorname{dim} B$ for which $\beta(\zeta)=\tilde{x}, a \zeta=j_{0}^{r}[x]$.

We have analogous definitions of the semi-holonomic and holonomic infinitesimal displacements. Thus if e.g. $\zeta$ is a holonomic infinitesimal displacement of $E_{x}$ then it is the $r$-jet of a differentiable section $u \rightarrow \eta_{u}$, where $\eta_{u}: E_{x} \rightarrow E_{f(u)}$ and $\eta_{x}$ is the identity of $E_{x}$.

Definition 2.2. ([5]) A non-holonomic element of connection of order $r$ at the point $x \in B$ is any element $X \in \tilde{J}_{x}^{r}(B, \Phi)$ for which $\beta(X)=\tilde{x}, a X=\hat{\jmath}_{x}^{r}$ and $b X=j_{x}^{r}$.

We define again analogously the semi-holonomic and holonomic elements of connection. Especially if $X$ is a holonomic element of connection of order $r$ at $x \in B$ then it is an $r$-jet at $x$ of a differentiable section $u \rightarrow \sigma_{u}$, where $\sigma_{u}: E_{x} \rightarrow E_{u}$ and $\sigma_{x}$ is the identity of $E_{x}$. Denote by $\widetilde{Q}^{r}=\widetilde{Q}^{r}\left(\Phi^{*}\right)$ or $\bar{Q}^{r}$ or $Q^{r}$ the manifold of all nonholonomic or semi-holonomic or holonomic elements of connection respectively related to the differentiable groupoid $\Phi^{\circ}$. Let us restrict ourselves in the next only to locally trivial differentiable groupoids $\Phi^{\bullet}$ which implies, according to lemma 1.1, that $E$ is a differentiable fibre bundle $E\left(B, F, G^{*}, H, \Phi^{*}\right)$. Then it can be shown (c.f. also [17] for $r=1$ ) that $\widetilde{Q}^{r}$ is a non-empty regularly imbedded submanifold of $\tilde{J}^{r}(B, \Phi)$ and $\alpha: \widetilde{Q}^{r} \rightarrow B$ is a differentiable projection of maximal rank, $\widetilde{\Phi}^{r} \bullet$ being a differentiable groupoid of operators on $\widetilde{Q}^{r}$. The action of $\tilde{\Phi}^{r \bullet}$ upon $\widetilde{Q}^{r}$ is defined by the formula $Y \in \widetilde{\Phi}^{r}, X \in \widetilde{Q}^{r}, \alpha(Y)=\alpha(X)=x \Rightarrow$

$$
Y \bullet X=\left(Y . X \cdot j_{x}^{r}[\beta(\sigma Y)]\right)(b Y)^{-1},
$$

and thus the projection of the associated fibred structure in $\widetilde{Q}^{r}$ coincides with the source mapping $\alpha: \widetilde{Q}^{r} \rightarrow B$. In fact we have for $Y^{\prime} \in \widetilde{\Phi}^{r}, \alpha\left(Y^{\prime}\right)=\beta(b Y)=y$ the relation $Y^{\prime} \bullet(Y \bullet X)=\left(Y^{\prime} b Y . Y \cdot j_{x}^{r}[\beta(\sigma Y)] \cdot j_{y}^{r}\left[\beta\left(\sigma Y^{\prime}\right)\right]\right)(b Y)^{-1}\left(b Y^{\prime}\right)^{-1}=\left(Y^{\prime} . Y\right.$. $\left.. X . j_{x}^{r}\left[\beta\left(\sigma Y . \sigma Y^{\prime}\right)\right]\right) b\left(Y^{\prime} \bullet Y\right)^{-1}=\left(Y^{\prime} \bullet Y\right) \bullet X$, the other properties being evident. One can treat analogously the spaces $\bar{Q}^{r}=\widetilde{Q}^{r} \cap \bar{J}^{r}(B, \Phi)$ and $Q^{r}=\widetilde{Q}^{r} \cap J^{r}(B, \Phi)$.

Remark. We shall show that $\widetilde{\Phi}^{r \bullet}$ need not be an effective groupoid of operators on $\widetilde{Q}^{r}$ even if $\Phi^{\bullet}$ operates effectively on $E$. Denoting now by $K=K\left(\widetilde{Q}^{r}\right)$ the kernel of the operation of $\widetilde{\Phi}^{r \bullet}$ on $\widetilde{Q}^{r}$ defined analogously as in (1.1) we have for each $S \in K$ the relation $b S=a S=j_{x}^{r}$ and $K$ consists of only holonomic jets. Let us find this kernel explicitly in the special case when $E$ is a vector bundle the structure group being $G^{*}=$ $=G L(m, R)^{\circ}$, where $m$ is the dimension of the fibre type $F$. Chosing local coordinates in $B$ and a local chart of $E$ we get from (2.1) for $S=j_{x}^{r} A \in K$ the relation

$$
j_{x}^{r}\left(\boldsymbol{A} \cdot \mathbf{B} \cdot \mathbf{A}(x)^{-1}\right)=j_{x}^{r} \mathbf{B},
$$


where $\boldsymbol{A}(u)$ and $\mathbf{B}(u)$ are matrices, $\mathbf{B}(x)$ is the unity matrix. Especially for $r=1$ we have from (2.2)

$$
\mathrm{d} \boldsymbol{A}(x) \cdot \boldsymbol{B}(x) \cdot \boldsymbol{A}(x)^{-1}+\mathbf{A}(x) \cdot \mathrm{d} \boldsymbol{B}(x) \cdot \boldsymbol{A}(x)^{-1}=\mathrm{d} \boldsymbol{B}(x) .
$$

Taking $\mathrm{d} \boldsymbol{B}(x)=0$ we get $\mathrm{d} \boldsymbol{A}(x)=0$ and also that $\boldsymbol{A}(x)$ commutes with all the matrices $\mathrm{d} \mathbf{B}(x)$ which implies that $\boldsymbol{A}(x)$ is necessarily diagonal. Thus $K$ contains elements $S=j_{x}^{1} A$, where $A(u): E_{u} \rightarrow E_{u}$ is a homothety of fibres the coefficient being independent of $u$. This is also an immediate consequence of (2.3). Especially $K$ is not trivial and $\Phi^{1 \bullet}$ is not an effective groupoid of operators on $Q^{1}$.

Since we have supposed, returning to the general case, the groupoid $\Phi^{\bullet}$ to be locally trivial, the same is true about $\tilde{\Phi}^{r \bullet}$ or $\bar{\Phi}^{r \bullet}$ or $\Phi^{r \bullet}$ (c.f. lemma 1.4) and thus $\widetilde{Q}^{r}$ or $\bar{Q}^{r}$ or $Q^{r}$ are fibre bundles with fibre types being identifiable with $\widetilde{T}_{n, e}^{r}(G)$ or $\bar{T}_{n, e}^{r}(G)$ or $T_{n, e}^{r}(G)$ respectively (c.f. [5]), where $\widetilde{T}_{n, e}^{r}(G)=\left\{f \in \widetilde{T}_{n}^{r}(G) \mid \beta(f)=e\right\}$ etc. In the non-holonomic case this identification is given as follows. If $X \in \widetilde{Q}^{r}, \alpha(X)=c$ and $\varphi, \Sigma$ are fixed as in (1.6), where we may suppose $\beta(\Sigma)=e$, we have for the corresponding $f \in \widetilde{T}_{n, e}^{r}(G)$ the formula

$$
f=(\sigma \Sigma . X) \varphi^{-1}
$$

It is not difficult to see that this gives a one-to-one correspondence. Since the structure group of $\widetilde{Q}^{r}$ can be identified with $\left(\tilde{L}_{n}^{r} \times \widetilde{T}_{n}^{r}(G)\right)^{\bullet}$ according to (1.6), we derive for $(y, S) \in \tilde{L}_{n}^{r} \times \tilde{T}_{n}^{r}(G)$ and $f \in \widetilde{T}_{n, e}^{r}(G)$ the composition

$$
(y, S) \bullet f=(S . f) y^{-1} \cdot j_{0}^{r}[\beta(\sigma S)] .
$$

In fact, we have $(y, S) \bullet f=(\sigma \Sigma .(Y \bullet X)) \varphi^{-1}=\left(\sigma \Sigma . Y . X \cdot j_{c}^{r}[\beta(\sigma Y)]\right)(b Y)^{-1}$. . $\varphi^{-1}=\sigma \Sigma \varphi^{-1} \cdot Y(b Y)^{-1} \varphi^{-1} \cdot \Sigma(b Y)^{-1} \varphi^{-1} \cdot \sigma \Sigma(b Y)^{-1} \varphi^{-1} \cdot X(b Y)^{-1} \varphi^{-1}$. . $j_{c}^{r}[\beta(\sigma Y)](b Y)^{-1} \varphi^{-1}=S .(\sigma \Sigma . X) \varphi^{-1} \varphi(b Y)^{-1} \varphi^{-1} \cdot j_{0}^{r}[\beta(\sigma S)]$.

We shall connect with the fibre bundle $\widetilde{Q}^{r}$ its inverse $\widetilde{Q}^{r *}=\widetilde{Q}^{r *}\left(\Phi^{*}\right) \subset \tilde{J}^{r}(B, \Phi)$ defined by the condition $X \in \widetilde{Q}^{r *} \Leftrightarrow \sigma X \in \widetilde{Q}^{r}$. The elements $X \in \widetilde{Q}^{r *}$ are called nonholonomic coelements of connection of order $r$ at the point $X=\alpha(X)$ and they satisfy $a X=j_{x}^{r}, b X=\hat{\jmath}_{x}^{r}$. The groupoid $\widetilde{\Phi}^{r \bullet}$ is again a differentiable groupoid of operators on $\widetilde{Q}^{r *}$ if we define for $Y \in \widetilde{\Phi}^{r}, X \in \widetilde{Q}^{r *}, \alpha(Y)=\alpha(X)=x$

$$
Y \bullet X=\sigma(Y \bullet \sigma X)=\left(j_{x}^{r}[\beta(Y)] . X . \sigma Y\right)(b Y)^{-1},
$$

(c.f. [17] for $r=1$ ). We have again the identification of the fibre type of $\widetilde{Q}^{r *}$ with $\widetilde{T}_{n, e}^{r}(G)$, however the correspondence (2.4) goes now into $f=(X . \Sigma) \varphi^{-1}$ and this gives

$$
(y, S) \bullet f=j_{0}^{r}[\beta(S)] \cdot(f . \sigma S) y^{-1},
$$

and analogously in the semi-holonomic and holonomic cases. Thus we have the 
Proposition 2.1. Let $\Phi^{\bullet}$ be a locally trivial differentiable groupoid of operators on the manifold $E$ which becomes in this way a fibre bundle $E=E\left(B, F, G{ }^{*}, H, \Phi^{*}\right)$. Then $\widetilde{Q}^{r}\left(\Phi^{\bullet}\right)=\widetilde{Q}^{r}\left(B, \widetilde{T}_{n, e}^{r}(G),\left(\tilde{L}_{n}^{r} \times \widetilde{T}_{n}^{r}(G)\right)^{\bullet}, \tilde{H}^{r}(B) 凶 \widetilde{D}^{r} H, \tilde{\Phi}^{r \bullet}\right)$ and $\widetilde{Q}^{r *}\left(\Phi^{\bullet}\right)=$ $=\widetilde{Q}^{r *}\left(B, \widetilde{T}_{n, e}^{r}(G),\left(\tilde{L}_{n}^{r} \times \widetilde{T}_{n}^{r}(G)\right) \bullet, \widetilde{H}^{r}(B) \llbracket \widetilde{D}^{r} H, \widetilde{\Phi}^{r \bullet}\right)$ are fibre bundles, the action of the structure group upon the fibre type being given by (2.5) and (2.7) respectively. Analogous results hold for $\bar{Q}^{r}, \bar{Q}^{r *}, Q^{r}, Q^{r *}$.

Let $\widetilde{T}_{n, \tilde{x}}^{r}(\Phi)=\left\{Z \in \widetilde{T}_{n}^{r}(\Phi), \beta(Z)=\tilde{x}\right\}$. One can assign to each element $X \in \widetilde{Q}^{r}$, $\alpha(X)=x$ an element $Z \in \widetilde{T}_{n, \tilde{x}}^{r}(\Phi)$, uniquely up to the operation of $\tilde{L}_{n}^{r}$, for which $a Z=$ $=j_{0}^{r}[x]$ and $b Z$ is inversible, i.e. an $n^{r}$-contact element of $\Phi$ with the origin $\tilde{x}$, zero horizontal projection $a$ and inversible vertical projection $b$. Conversely, each such contact element defineds uniquely an element of $\widetilde{Q}^{r}$. Denoting by $\widetilde{P}_{n}^{r}(\Phi)_{v}$ the set of those contact elements, we have the one-to-one correspondence $X \in \widetilde{Q}^{r} \leftrightarrow X \tilde{H}^{r}(B)_{\alpha(X)} \in$ $\in \widetilde{P}_{n}^{r}(\Phi)_{v}$. Especially $\widetilde{P}_{n}^{r}(\Phi)_{v}$ is a differentiable manifold with the differentiable groupoid of operators $\tilde{\Phi}^{r \bullet}$ acting upon it according to $Y \in \widetilde{\Phi}^{r \bullet}, X \tilde{H}^{r}(B)_{\alpha(X)} \in \widetilde{P}_{n}^{r}(\Phi)_{v}, \alpha(Y)=$ $=\alpha(X) \Rightarrow$

$$
Y \bullet X \tilde{H}^{r}(B)_{\alpha(X)}=(Y \bullet X) \tilde{H}^{r}(B)_{\alpha(X)} \text {. }
$$

Thus $\widetilde{P}_{n}^{r}(\Phi)_{v}$ becomes a fibre bundle $\widetilde{P}_{n}^{r}(\Phi)_{v}\left(B, \widetilde{T}_{n, e}^{r}(G) \tilde{L}_{n}^{r},\left(\tilde{L}_{n}^{r} \times \widetilde{T}_{n}^{r}(G)\right)^{\bullet}, \tilde{H}^{r}(B) 区\right.$ 凶 $\left.\tilde{D}^{r} H, \tilde{\Phi}^{r \bullet}\right)$. Analogously there is an isomorphism between the fibre bundles $\widetilde{Q}^{r *}$ and $\tilde{P}_{n}^{r}(\Phi)_{h}\left(B, \widetilde{T}_{n, e}^{r}(G) \tilde{L}_{n}^{r},\left(\tilde{L}_{n}^{r} \times \tilde{T}_{n}^{r}(G)\right)^{\bullet}, \quad \tilde{H}^{r}(B) 凶 \tilde{\mathscr{D}}^{r} H, \tilde{\Phi}^{r \bullet}\right)$, where $\tilde{P}_{n}^{r}(\Phi)_{h}=$ $=\left\{Z \in \widetilde{T}_{n, \tilde{x}}^{r}(\Phi) / \widetilde{L}_{n}^{r} \mid x \in B, b Z=j_{0}^{r}[x], a Z\right.$ inversible $\}$. Thus we get a commutative diagram

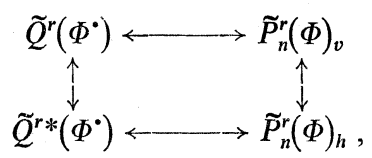

where in the rows there are canonical isomorphisms and in the columns canonical antiisomorphisms of fibre bundles with the basis $B$, their structure group being induced by the operation of $\tilde{\Phi}^{r \bullet}$. Analogous results are again obtained in the semiholonomic and holonomic cases.

Definition 2.3. ([5]) A non-holonomic (accordingly semi-holonomic and holonomic) connection of order $r$ in the locally trivial differentiable groupoid $\Phi^{\bullet}$ is given by a differentiable section $B \rightarrow \widetilde{Q}^{r}\left(\Phi^{*}\right)$ (accordingly $B \rightarrow \bar{Q}^{r}\left(\Phi^{*}\right)$ and $B \rightarrow Q^{r}\left(\Phi^{\bullet}\right)$ ), or, equivalently, by a differentiable section $B \rightarrow \widetilde{Q}^{r *}\left(\Phi^{\bullet}\right)$ (accordingly $B \rightarrow \bar{Q}^{r *}\left(\Phi^{\bullet}\right)$ and $B \rightarrow Q^{r *}\left(\Phi^{\bullet}\right)$ ), or by a differentiable section $B \rightarrow \widetilde{P}_{n}^{r}(\Phi)_{v}$ (accordingly $B \rightarrow \bar{P}_{n}^{r}(\Phi)_{v}$ and $\left.B \rightarrow P_{n}^{r}(\Phi)_{v}\right)$, or by a differentiable section $B \rightarrow \widetilde{P}_{n}^{r}(\Phi)_{h}\left(\right.$ accordingly $B \rightarrow \bar{P}_{n}^{r}(\Phi)_{h}$ and $\left.B \rightarrow P_{n}^{r}(\Phi)_{h}\right)$.

Note that a non-holonomic element of connection of order $r$ at the point $x \in B$ gives rise to a correspondence which assignes to each non-holonomic velocity of order $r$ in $B$ with origin $x \in B$ a non-holonomic infinitesimal displacement of order $r$ 
of the fibre $E_{x}$. Consequently a non-holonomic connection of order $r$ gives rise to a differentiable mapping of the manifold of non-holonomic velocities of order $r$ in $B$ into the manifold of all non-holonomic infinitesimal displacements of order $r$ of fibres of $E$, the fibre being taken at the origin of the mapped velocity. However, conversely each such differentiable mapping does not define in general a nonholonomic connection in $\Phi^{\bullet}$ in the sense of Ehresmann. It defines always a $\left(\mathscr{E}, T_{1}^{r}\right)$ connection in $\Phi^{\bullet}$ (c.f. ch. 3) which need not be a connection in the sense of Ehresmann. Analogously in the other two cases.

A non-holonomic or semi-holonomic or holonomic connection of order $r$ on the fibre bundle $E\left(B, F, G^{*}, H, \Phi^{*}\right)$ is a non-holonomic or semi-holonomic or holonomic connection of order $r$ respectively in the associated groupoid $\Phi^{\bullet}$.

It can be seen, e.g. from the results of the following chapter, that if $r=1$ and the groupoid $\Phi^{\bullet}$ operates effectively on $E$ (i.e. $G^{\bullet}$ operates effectively on $F$ ) this definition of a connection on the fibre bundle $E\left(B, F, G^{*}, H, \Phi^{*}\right)$ coincides with the one defined in [14]. On the other hand in general denoting by $K^{\bullet} \subset \Phi^{\bullet}$ the kernel of the operation as in (1.1) we see that $j^{r} \Pi: \tilde{J}^{r}(B, \Phi) \rightarrow \tilde{J}^{r}(B, \Phi / K)$, where $\Pi: \Phi \rightarrow \Phi / K$ is the natural projection, takes any connection in $\Phi^{\bullet}$ into a connection in the associated effective groupoid of operators $(\Phi / K)^{\circ}$. Conversely, $X, X^{\prime} \in \widetilde{Q}^{r}\left(\Phi^{\bullet}\right)$ are projected into the same element of connection with respect to the associated effective groupoid $(\Phi / K)^{\circ}$ iff $X^{\prime}=X$. $\xi$, where $\xi \in \tilde{J}^{r}(B, K), \alpha(\xi)=x, \beta(\xi)=\tilde{x}$ and $a \xi=b \xi=\hat{\jmath}_{x}^{r}$ (c.f. (1.12)).

It has been shown in [5] that if $\Phi^{\bullet}$ is a locally trivial differentiable groupoid of operators on the manifold $E$ then a connection of any order and any type always exists. Note that if $\Phi^{\bullet}$ is not locally trivial the $\widetilde{Q}^{r}\left(\Phi^{\bullet}\right)$ may be empty. E.g. if $K^{\bullet}$ is the stable subgroupoid of a differentiable groupoid of operators defined above in (1.1) we see that it is itself a differentiable groupoid of operators (however, not even transitive), but $\widetilde{Q}^{r}\left(K^{*}\right)$ is empty.

We have given here four equivalent characterizations of higher order connections on a fibre bundle $E$. We see that the connection depends essentially upon the principal fibre bundle associated with $E$ and in fact, one can define a higher order connection applying only the principal fibre bundle (c.f. [1], [2]) as it is the case with $r=1$ e.g. in [14]. We shall obtain these characterizations as special cases of the results of the following chapter.

In order to accomplish these introductory parts we give now the definition of the projection and prolongation of a higher order connection as introduced by Ehresmann in [5] (c.f. also [1]) in a more detailed notation.

Thus let again $\Phi^{\bullet}$ be a locally trivial differentiable groupoid. Denote by $\pi$ : $: \tilde{J}^{r}(B, \Phi) \rightarrow \tilde{J}^{r-1}(B, \Phi)$ the natural projection. Its restriction defines a bundle projection $\pi_{Q}: \widetilde{Q}^{r} \rightarrow \widetilde{Q}^{r-1}$. In fact, if $X \in \widetilde{Q}^{r}$ we have $\alpha(\pi X)=\alpha(X), \beta(\pi X)=\pi \beta(X)=$ $=\tilde{\alpha}(X), a \pi X=\pi a X=\hat{j}_{\alpha(X)}^{r-1}$ and $b \pi X=j_{\alpha(X)}^{r-1}$. Especially if $X: B \rightarrow \widetilde{Q}^{r}$ is a nonholonomic connection of order $r$ then the non-holonomic connection of order $r-1$ $\pi_{Q} X: B \rightarrow \widetilde{Q}^{r-1}$ is called the projection of $X$. Thus a non-holonomic colınection of order $r$ defines by subsequent projections a sequence of connections of orders one to 
$r-1$. If the starting connection is semi-holonomic then this sequence consists of semi-holonomic connections and if it is a holonomic connection the sequence consists of holonomic ones.

Conversely, given a non-holonomic connection $X: B \rightarrow \widetilde{Q}^{r}$ we define its prolongation, which is a non-holonomic connection of order $r+1$, as follows. Denote by $X_{1}: B \rightarrow Q^{1}$ the projection of $X$. If $u \in B$ denote by $v \rightarrow \xi_{u}(v)$ the local mapping from $B$ into $\Phi$ such that $X_{1}(u)=j_{u}^{1} \xi_{u}$. Note that $\xi_{u}(u)=\tilde{u}$, and $j_{u}^{1}\left(v \rightarrow a \xi_{u}(v)\right)=$ $=\hat{\jmath}_{u}^{1}, \quad j_{u}^{1}\left(v \rightarrow b \xi_{u}(v)\right)=j_{u}^{1}$ imply $j_{u}^{1}\left(v \rightarrow j_{v}^{r}\left[a \xi_{u}(v)\right]\right)=j_{u}^{1}\left(v \rightarrow j_{v}^{r}[u]\right)$ and $j_{u}^{1}(v \rightarrow$ $\left.\rightarrow j_{v}^{r}\left[b \xi_{u}(v)\right]\right)=j_{u}^{1}\left(v \rightarrow j_{v}^{r}[v]\right)$. Define

$$
X^{\prime}(u)=j_{u}^{1} X \cdot j_{u}^{1}\left(v \rightarrow j_{v}^{r}\left[\xi_{u}(v)\right]\right) \in \tilde{J}^{r+1}(B, \Phi) .
$$

The composition here is really defined since $a j_{u}^{1} X=j_{u}^{1} a X=j_{u}^{1}\left(v \rightarrow \hat{\jmath}_{v}^{r}\right)$ and $b j_{u}^{1}(v \rightarrow$ $\rightarrow j_{v}^{r}\left[\xi_{u}(v)\right]=j_{u}^{1}\left(v \rightarrow j_{v}^{r}\left[b \xi_{u}(v)\right]\right)=j_{u}^{1}\left(v \rightarrow \hat{\jmath}_{v}^{r}\right)$. We have $X^{\prime}(u) \in \widetilde{Q}^{r+1}$. In fact $u \rightarrow$ $\rightarrow X^{\prime}(u)$ is clearly differentiable and $\alpha\left(X^{\prime}(u)\right)=u, \beta\left(X^{\prime}(u)\right)=\tilde{u} \cdot j_{u}^{r}\left[\xi_{u}(u)\right]=\tilde{u}$, $a X^{\prime}(u)=a j_{u}^{1}\left(v \rightarrow j_{v}^{r}\left[\xi_{u}(v)\right]\right)=j_{u}^{1}\left(v \rightarrow a j_{v}^{r}\left[\xi_{u}(v)\right]\right)=j_{u}^{1}\left(v \rightarrow j_{v}^{r}\left[a \xi_{u}(v)\right]=\hat{\jmath}_{u}^{r+1}\right.$ and $b X^{\prime}(u)=j_{u}^{1} b X=j_{u}^{r+1}$.

Thus (2.10) defines the prolongation $X^{\prime}$ of the non-holonomic connection $X$. Note that $\pi_{Q} X^{\prime}=X$ follows immediately from (2.10). Especially let $X: B \rightarrow Q^{1}$ be a first order connection in $\Phi^{\bullet}$ and define recurrently $X_{1}=X$ and $X_{r}$ let be the prolongation of the connection $X_{r-1}, r>1$. From (2.10) we obtain the recurrent formula

$$
X_{r}(u)=j_{u}^{1}\left(v \rightarrow X_{r-1}(v) \cdot j_{v}^{r-1}\left[\xi_{u}(v)\right]\right),
$$

where $X(u)=j_{u}^{1} \xi_{u}$, i.e. $\xi_{u}(v) \in \Phi$ for $v$ in a neighbourhood of $u \in B$. We call the members of this sequence $\left\{X_{r}\right\}_{r=1}^{\infty}$ simple connections in $\Phi^{\bullet}$ generated by $X$.

Proposition 2.1. ([5]) If $\Phi^{\bullet}$ is a locally trivial differentiable groupoid then each simple connection in it generated by a first order connection $X_{1}=X: B \rightarrow Q^{1}$ is a semi-holonomic connection.

Proof. Note that an element $Y(u) \in \tilde{J}^{r}\left(V_{n}, V_{m}\right), u \in V_{n}, \alpha Y(u)=u$ is semiholonomic by definition iff $Y(u)=j_{u}^{1} \sigma_{u}=j_{u}^{1}\left(v \rightarrow \sigma_{u}(v)\right)$, where $\sigma_{u}(v)$ is semi-holonomic for all $v$ in a neighbourhood of $u$ and

$$
j_{u}^{1}\left(v \rightarrow\left(\pi \sigma_{u}\right)(v)\right)=\sigma_{u}(u) .
$$

Now it is easy to prove by induction that if $r \geqq 1$ then each $X_{r}(u), u \in B$ is semiholonomic. Suppose namely that $X_{r}(u), r \geqq 1$ is semi-holonomic for all $u \in B$ and write $X_{r+1}(u)=j_{: z}^{1} \sigma_{u}$. We see from the definition (2.11) of $X_{r+1}(u)$ that $v \rightarrow \sigma_{u}(v)$ is a semi-holonomic section and also that the relation (2.12) to prove follows from the same formula since $\pi X_{r+1}(v)=X_{r}(v)$ and $\xi_{u}(u)=\tilde{u}$. Thus also $X_{r+1}(u)$ is semi-holonomic for all $u \in B$ and this completes the proof.

Finally let us make the following remark. If $\Phi^{\bullet}$ is a differentiable groupoid of operators on the manifold $E$ such that $\Phi_{E}^{\cdot}$ is locally trivial one may construct the fibre 
bundle $\widetilde{Q}^{r}\left(\Phi_{E}^{\cdot}\right)$ which can be canonically identified with the subspace of $\tilde{J}^{r}(E, \Phi)$ consisting of such elements $X \in \tilde{J}^{r}(E, \Phi), \alpha(X)=z$ for which $a X=j_{z}^{r}[p(z)]$ and $X . \hat{j}_{z}^{r}=j_{z}^{r}$. The corresponding associated groupoid of operators having been identified in chapter 1 with the subspace of such $Y \in \tilde{J}^{r}(E, \Phi)$ for which $a Y=j_{\alpha(Y)}^{r} p$ and $Y . j_{\alpha(Y)}^{r} \in \widetilde{\Pi}^{r} E$, we get the composition rule $Y \in \tilde{\Phi}_{E}^{r}, X \in \widetilde{Q}^{r}\left(\Phi_{E}^{*}\right), z=\alpha(Y)=\alpha(X) \Rightarrow$

$$
Y \bullet X=\left(Y \cdot X \cdot j_{z}^{r}[\beta(\sigma Y)]\right)\left(Y \cdot j_{z}^{r}\right)^{-1} \text {, }
$$

which is well defined and can be obtained as a special case from the general composition rule (2.1).

\section{3. $(\mathscr{V}, \mathscr{T})$-CONNECTIONS ON FIBRE BUNDLES}

Denote by $\mathscr{C}$ the category of differentiable manifolds (including dimension zero), and by $\left.\mathscr{E}^{3}\right)$ the category of fibred manifolds. Objects in $\mathscr{E}$ are pairs $(B, C)$ of differentiable manifolds (identified with the pairs of identities on them) with a differentiable mapping $p_{B}=p: C \rightarrow B$ which is onto, and morphisms in $\mathscr{E}$ are pairs $(f, F)$ : $:\left(B_{1}, C_{1}\right) \rightarrow\left(B_{2}, C_{2}\right)$ of differentiable mappings such that $p F=f p$. Further denote by $\mathscr{E}^{0}$ the subcategory of $\mathscr{E}$ consisting of all locally trivial fibred manifolds with a Lie structure group, i.e. of all fibre bundles. This is a complete subcategory. Finally denote by $\mathscr{E}_{L} \subset \mathscr{E}^{0}$ the subcategory of all vector bundles, $(B, C)$ being a vector bundle iff the structure group is a subgroup of a linear group. In this case the mappings $F$ are supposed linear on each fibre of $\left(B_{1}, C_{1}\right)$.

Denote by $\tilde{J}^{r}$ the category of differentiable sections in all the fibre bundles $\tilde{J}^{r}\left(V_{n}, V_{m}\right)$ with basis $V_{n}$. The objects or unities are differentiable manifolds or $r$-flots of identities on these manifolds. Let further $\hat{J}^{r} \in \tilde{J}^{r}$ denote any section $x \rightarrow j_{x}^{r}[x]$. Thus each $\hat{J}^{r}$ is a section in $\tilde{J}^{r}\left(V_{n}, V_{n}\right)$. One defines analogously the subcategories $J^{r} \subset \bar{J}^{r}$ of $\tilde{J}^{r}$.

Definition 3.1. A connector $\mathscr{T}$ is a covariant functor from the category $\mathscr{C}$ into the category $\mathscr{E}$ with the following properties:

(T1) $\mathscr{T}$ assigns to each differentiable mapping $f$ a morphism $(f, \mathscr{T}(f))$ of $\mathscr{E}$, especially to the manifold $B$ a fibred manifold $(B, \mathscr{T}(B))$;

(T2) there exists for each differentiable manifold $B$ a differentiable injection $\iota_{B}=$ $=\iota: B \rightarrow \mathscr{T}(B)$ such that $p \iota=\mathrm{id}_{B}$, and if $f$ is a differentiable mapping then $\mathscr{T}(f) \iota=\imath f$

(T3) if $f$ is a differentiable mapping which is injective of maximal rank (i.e. inducing an injection of the corresponding tangent bundles) then the same is true about $\mathscr{T}(f)$;

3) For the sake of simplicity we omit the composition symbol in the notation of the categories $\mathscr{C}, \mathscr{E}$ and their subcategories since the composition is here evident. 
(T4) if $B$ consists of only one point, $B=\{c\}$ then the same holds about $\mathscr{T}(B)$, i.e. $\mathscr{T}(B)=\{\iota c\}$.

We say that the connector $\mathscr{T}$ is into $\mathscr{V}$ if $\mathscr{V}$ is a subcategory of $\mathscr{E}$ and $\mathscr{T}$ takes values in $\mathscr{V}$.

First note that if $\mathscr{T}$ is into $\mathscr{V} \subset \mathscr{E}^{0}$ then (T2) implies that each $\iota_{B}$ is injective of maximal rank. According to (T3) if $B_{1}$ is a submanifold injected and regularly imbedded into $B_{2}$ then we can suppose also $\mathscr{T}\left(B_{1}\right) \subset \mathscr{T}\left(B_{2}\right)$, i.e. denoting by $j: B_{1} \rightarrow B_{2}$ this inclusion we identify $\mathscr{T}\left(B_{1}\right)$ with $\mathscr{T}(j) \mathscr{T}\left(B_{1}\right) \subset \mathscr{T}\left(B_{2}\right)$. Note that this identification takes $p_{B_{1}}$ into the restriction of $p_{B_{2}}$ and $\iota_{B_{1}}$ into the rectriction of $\iota_{B_{2}}$ (c.f. (T1) and (T2)), and if $f: B_{2} \rightarrow B_{2}^{\prime}$ is a differentiable mapping hen $\mathscr{T}\left(\left.f\right|_{B_{1}}\right)=\left.\mathscr{T}(f)\right|_{\mathscr{T}\left(B_{1}\right)}$ since $\left.f\right|_{B_{1}}=f j$ implies $\mathscr{T}\left(\left.f\right|_{B_{1}}\right)=\mathscr{T}(f) \mathscr{T}(j)$. To simplify the notations this convention is employed throughout the paper. It justifies the following

Lemma 3.1. (c.f. lemma 1.3). Let $B_{1}$ be a submanifold injected and regularly imbedded into the differentiable manifold $B_{2}$. Let $B_{2}^{\prime}$ be another differentiable manifold and $f, f^{\prime}: B_{2} \rightarrow B_{2}^{\prime}$ differentiable mappings such that $x \in B_{1} \Leftrightarrow f x=f^{\prime} x$, and let $\mathscr{T}$ be a connector. Then $X \in \mathscr{T}\left(B_{1}\right) \Leftrightarrow \mathscr{T}(f) X=\mathscr{T}\left(f^{\prime}\right) X$.

Proof. In fact, with the above notations, $f j=f^{\prime} j$ implies $\mathscr{T}(f) \mathscr{T}(j)=\mathscr{T}\left(f^{\prime}\right) \mathscr{T}(j)$.

Lemma 3.2. If $\mathscr{T}$ is a connector, then $\mathscr{T}[k]=[\iota k]$, i.e. $\mathscr{T}$ takes constant mappings into constant mappings.

Proof. We have $[k]: B_{1} \rightarrow B_{2}, k \in B_{2} \Rightarrow \mathscr{T}\{k\} \subset \mathscr{T}\left(B_{2}\right)$. According to (T4), $\mathscr{T}\{k\}$ consists of only one point $\{l k\}$ and thus $\mathscr{T}[k]: \mathscr{T}\left(B_{1}\right) \rightarrow\{l k\} \subset \mathscr{T}\left(B_{2}\right)$ must be of the form $[\iota k]$.

Definition 3.2. A connector $\mathscr{T}$ is called local if $U \subset B$ open implies $\mathscr{T}(U)=$ $=\{X \in \mathscr{T}(B) \mid p X \in U\}$.

Lemma 3.3. Let $f: B_{1} \rightarrow B_{2}$ be differentiable and let there exist an open covering of $B_{2}$ such that for each $U$ of this covering there exists a differentiable mapping $g_{U}: U \rightarrow B_{1}$ satisfying $f g_{U}=\mathrm{id}_{U}$. Then $\mathscr{T}$ being a local connector, $\mathscr{T}(f): \mathscr{T}\left(B_{1}\right) \rightarrow$ $\rightarrow \mathscr{T}\left(B_{2}\right)$ is a projection of maximal rank.

Proof. We have $\mathscr{T}(f) \mathscr{T}\left(g_{U}\right)=\operatorname{id}_{\mathscr{T}(U)}$ hence each $\mathscr{T}(U)$ belongs to $\operatorname{Im} \mathscr{T}(f)$ and since the $\mathscr{T}(U)$ cover $\mathscr{T}\left(B_{2}\right), \mathscr{T}(f)$ is onto.

Lemma 3.4. If $\mathscr{T}$ is a connector into $\mathscr{E}_{L}$ then $\iota x$ is the zero element in $\mathscr{T}(B)_{x}$ for each differentiable manifold $B$ and each $x \in B$.

Proof. Take $B_{1}=\{c\}$, i.e. $\operatorname{dim} B_{1}=0$. Then $\iota c$ is the zero element in $\mathscr{T}\left(B_{1}\right)$. If now $f: B_{1} \rightarrow B$ is such that $f(c)=x$ we have $\iota x=\iota f(c)=\mathscr{T}(f) \iota c$ and this is again a zero element since $\mathscr{T}(f)$ is linear on $\mathscr{T}\left(B_{1}\right)$. 
Now we are passing to the most general definition of a connection on a fibre bundle or, as a matter of fact, in a locally trivial differentiable groupoid. As in the preceding denote by $E$ any fibre bundle with the basis $B$, fibre type $F$, structure group $G^{\bullet}$ and associated principal bundle $H$. Let $\Phi^{\bullet}=H H^{-1}$ be its associated groupoid.

Definition 3.3. Let $E=E\left(B, F, G^{*}, H, \Phi^{*}\right)$ be a fibre bundle and let $\mathscr{T}$ be a connector into $\mathscr{V} \subset \mathscr{E}$. A $(\mathscr{V}, \mathscr{T})$-connection on $E$ is a mapping $\mathscr{X}: \mathscr{T}(B) \rightarrow \mathscr{T}(\Phi)$ such that

$$
\begin{gathered}
(\sim, \mathscr{X}) \text { belongs to } \mathscr{V} ; \\
\mathscr{T}(b) X=\operatorname{id}_{\mathscr{T}(B)} ; \\
\mathscr{T}(a) \mathscr{X}=\iota p
\end{gathered}
$$

where $a, b: \Phi \rightarrow B$ have the usual meanings.

First note that if $\mathscr{V} \subset \mathscr{V}^{\prime} \subset \mathscr{E}$ then each $(\mathscr{V}, \mathscr{T})$-connection is a $\left(\mathscr{V}^{\prime}, \mathscr{T}\right)$-connection and if $\mathscr{V} \subset \mathscr{V}^{\prime}$ is a complete subcategory and $\mathscr{T}$ is into $\mathscr{V}$ then each $\left(\mathscr{V}^{\prime}, \mathscr{T}\right)$ connection is a $(\mathscr{V}, \mathscr{T})$-connection. Thus one could restrict himself to the case when all the objects in $\mathscr{V}$ are of the form $(B, \mathscr{T}(B))$.

Theorem 3.1. Let there exist an isomorphism $(i, I)$ of the category $\tilde{J}^{r}\left(\right.$ or $\bar{J}^{r}$, or $\left.J^{r}\right)$ onto the category $\mathscr{V}$ satisfying

$$
\begin{aligned}
& i(Z) x=\beta\left(Z_{x}\right) ; \\
& I\left(j^{r} f\right)=\mathscr{T}(f) ; \\
& I\left(\hat{J}^{r}\right)=\iota p,
\end{aligned}
$$

where $Z: x \rightarrow Z_{x}$ is a differentiable section in some $\tilde{J}^{r}\left(V_{n}, V_{m}\right)$ (or $\bar{J}^{r}\left(V_{n}, V_{m}\right)$, or $\left.J^{r}\left(V_{n}, V_{m}\right)\right)$. Then there is a one-to-one correspondence between $(\mathscr{V}, \mathscr{T})$-connections and non-holonomic (or semi-holonomic, or holonomic) connections of order $r$ in the sense of Ch. Ehresmann (c.f. definition 2.3).

Proof. We shall deal only with the non-holonomic case. A $(\mathscr{V}, \mathscr{T})$-connection determines uniquely a section $Z$ in $\tilde{J}^{r}(B, \Phi)$. From $(3.1)$ and $(C 1)$ we get $\beta\left(Z_{x}\right)=\tilde{x}$. Since $\mathscr{X}=I(Z)$ we get from the covariance of $(i, I)$ and from (3.2) the transformation of (C2), (C3) into $b Z=I^{-1}\left(\mathrm{id}_{\mathscr{T}(B)}\right), a Z=I^{-1}(\iota p)$ respectively and hence according to (3.3) the required conditions for $Z \in \widetilde{Q}^{r}$. Conversely let $Z$ be a section in $\widetilde{Q}^{r} \subset$ $\subset \tilde{J}^{r}(B, \Phi)$. Then $i(Z) x=\tilde{x}$ because of $(3.1)$ and $\beta\left(Z_{x}\right)=\tilde{x}$. Thus $(\sim, I(Z)) \in \mathscr{V}$. The relations (C2), (C3) are established analogously as in the above case.

Now we are going to give some concrete examples of connectors and $(\mathscr{V}, \mathscr{T})$ connections which will not only serve as an illustration to the general theory developped in the sequel, but also give rise to several equivalent formulations of the definition 
of higher order connections, as contained in [1], [2], [12], from a unified point of view.

Define the local connector $\tilde{J}_{s}^{r}\left(V_{p},.\right)$ as follows. $V_{p}$ is a fixed differentiable manifold and $s$ is a fixed point in it. The assignement is given by $B \rightarrow\left(B, \tilde{J}_{s}^{r}\left(V_{p}, B\right)\right)$ and $\tilde{J}_{s}^{r}\left(V_{p}, f\right) Z=j^{r} f Z$, where in the last term we have the composition of jets. The projection $p$ is in this case the target mapping $\beta$ and for $x \in B$ we define $x=$ $=j_{s}^{r}[x] \in \tilde{J}_{s}^{r}\left(V_{p}, B\right)$. It is not difficult to see that this is really a local connector. In fact $\tilde{J}_{s}^{r}\left(V_{p}, f\right) \iota x=j^{r} f j_{s}^{r}[x]=j_{s}^{r}[f(x)]=\iota f(x)$ and (T3) follows from the local expression for the composition of jets (c.f. lemma 1.2), the other properties being evident. Analogously one defines $\bar{J}_{s}^{r}\left(V_{p},.\right)$ and $J_{s}^{r}\left(V_{p},.\right)$.

If $E$ is a fibre bundle we can define a $\left(\mathscr{E}, \tilde{J}_{s}^{r}\left(V_{p},.\right)\right)$-connection in it which generalizes the notion of a non-holonomic connection of order $r$. Since $\mathscr{E}$ does not satisfy the assumptions of theorem 3.1, we need to restrict the category $\mathscr{E}$ in order to obtain the Ehresmann's higher order connections. For this purpose we connect with each $\tilde{J}_{s}^{r}\left(V_{p},.\right)$ a category denoted by $\tilde{C}_{s}^{r}\left(V_{p},.\right)$, where the objects are exactly all the fibre bundles $\left(B, \tilde{J}_{s}^{r}\left(V_{p}, B\right)\right)$ and morphisms are all those of the form $(f, F)$ with $F$ : $: \tilde{J}_{s}^{r}\left(V_{p}, B_{1}\right) \rightarrow \tilde{J}_{s}^{r}\left(V_{p}, B_{2}\right)$ given by $F(X)=Z_{\beta(X)} X$, where $x \rightarrow Z_{x}$ is a differentiable section in $\tilde{J}^{r}\left(B_{1}, B_{2}\right)$ and $f(x)=\beta\left(Z_{x}\right)$. Denoting $i(Z)=f$ and $I(Z)=F$ we see at once that theorem 3.1 applies to $\widetilde{C}_{s}^{r}\left(V_{p},.\right)$. We get again the same definitions and results in the semi-holonomic and holonomic cases.

We call a jet $Z \in \tilde{J}^{r}\left(B_{1}, B_{2}\right)$ injective if the relation $Y \in \tilde{J}^{r}\left(B, B_{1}\right) \rightarrow Z Y \in \tilde{J}^{r}\left(B, B_{2}\right)$ is injective. If this holds for some $B$ then it holds for each differentiable manifold. In fact, suppose $Z Y=Z Y^{\prime}$, where $Y, Y^{\prime} \in \tilde{J}^{r}\left(B^{\prime}, B_{1}\right)$. We have then for each $X \in$ $\in \tilde{J}^{r}\left(B, B^{\prime}\right), \beta(X)=\alpha(Y)$, the relation $Z Y X=Z Y^{\prime} X$ which implies $Y X=Y^{\prime} X$, but this means $Y=Y^{\prime}$. Note that if each $Z_{x}$ defined above is injective then the corresponding $(f, F)$ is an injection in the category $\widetilde{C}_{s}^{r}\left(V_{p},.\right)$. One could also give a charaterization of injective jets by means of their local expressions.

We write also $\tilde{T}_{k}^{r}$ instead of $\tilde{J}_{0}^{r}\left(R^{k},.\right)$ and analogously in the semi-holonomic and holonomic cases. Thus elements of $\widetilde{T}_{k}^{r}(B)$ are non-holonomic $k^{r}$-velocities on $B$.

Denote by $\widetilde{T}_{r, k}(B)$ the vector bundle over $B$ of $k^{r}$-vectors on $B$, i.e. the vector bundle dual to the vector bundle of $k^{r}$-covelocities (non-holonomic), $\widetilde{T}_{r, k}(B)_{x}=\widetilde{T}_{k}^{r *}(B)_{x}^{*}$ and $\tilde{T}_{k}^{r *}(B)_{x}=\left\{\xi \in \tilde{J}_{x}^{r}\left(B, R^{k}\right) \mid \beta(\xi)=0\right\}$. This defines a local connector assigning to $B$ the vector bundle $\left(B, \widetilde{T}_{r, k}(B)\right)$ and to the differentiable mapping $f$ the mapping $\left(f, \widetilde{T}_{r, k}(f)\right)$ defined by $\left\langle\widetilde{T}_{r, k}(f) Z, \xi\right\rangle=\left\langle Z, \xi j_{x}^{r} f\right\rangle, p(Z)=x, \alpha(\xi)=f(x), \iota x$ being defined as the zero element in $\widetilde{T}_{r, k}(B)_{x}$. We shall show explicitly only (T3). Thus suppose $\left\langle Z, \xi j_{x}^{r} f\right\rangle=\left\langle Z^{\prime}, \xi j_{x}^{r} f\right\rangle$ for all $\xi$ with $\alpha(\xi)=f(x)$, where $f$ is injective of maximal rank. This means that there exists a $g$ defined in a neighbourhood of $f(x)$ such that $g f=\mathrm{id}$ in a neighbourhood of $x$ and thus $j_{f(x)}^{r} g j_{x}^{r} f=j_{x}^{r}$. Consequently if $\xi^{\prime}$ is any element of $\widetilde{T}_{k}^{r *}(B)_{x}$ we have $\xi^{\prime}=\xi^{\prime} j_{f(x)}^{r} g j_{x}^{r} f$ and hence $\left\langle Z, \xi^{\prime}\right\rangle=\left\langle Z^{\prime}, \xi^{\prime}\right\rangle$. This implies $Z=Z^{\prime}$, i.e. $\widetilde{T}_{r, k}(f)$ is injective. More generally, if $A$ is a fixed vector space, denote by $\widetilde{T}_{r, k}(B, A)$ the vector bundle of all linear mappings from $\widetilde{T}_{k}^{r *}(B)_{x}$, $x \in B$ into $A$. This gives rise (at least if $A$ is of finite dimension) to local connectors 
$\widetilde{T}_{r, k}(., A)$. The local connectors $\bar{T}_{r, k}(., A)$ and $T_{r, k}(., A)$ are defined analogously.

We connect again with each $\widetilde{T}_{r, k}(., A)$ a category $\widetilde{C}_{r, k}(., A)$, where the objects are exactly all the vector bundles $\left(B, \widetilde{T}_{r, k}(B, A)\right)$ and morphisms are all those of the form $(f, F)$ with $F: \widetilde{T}_{r, k}\left(B_{1}, A\right) \rightarrow \widetilde{T}_{r, k}\left(B_{2}, A\right)$ given by $\langle F(X), \xi\rangle=\left\langle X, \xi Z_{x}\right\rangle$, where $x \rightarrow Z_{x}$ is a differentiable section in $\tilde{J}^{r}\left(B_{1}, B_{2}\right)$ and $f(x)=\beta\left(Z_{x}\right)$. Now theorem 3.1 applies again to $\widetilde{C}_{r, k}(., A)$. Analogously in the semi-holonomic and holonomic cases.

Lemma 3.5. Each linear mapping $J_{x}^{1}\left(B_{1}, R^{k}\right) \rightarrow J_{y}^{1}\left(B_{2}, R^{k}\right)$ preserving the targets of jets can be uniquely expressed as $\xi \rightarrow \xi X$, where $X \in J_{y}^{1}\left(B_{2}, B_{1}\right), \beta(X)=x$.

Proof. In fact, we get in a fixed system of coordinates $\xi_{i}^{h} \rightarrow \eta_{j}^{h}=\xi_{i}^{h} X_{j}^{i}$ and $X_{j}^{i}$ are thus the components of the required $X$.

This lemma shows that there is a natural isomorphism between the connectors $T_{k}^{1}$ and $T_{1, k}\left(., R^{k *} \otimes R^{k}\right)$ since each element of $T_{1, k}\left(B, R^{k *} \otimes R^{k}\right)$ can be interpreted as a linear mapping $J^{1}\left(B, R^{k}\right) \rightarrow J_{0}^{1}\left(R^{k}, R^{k}\right)$ preserving the zero target in $R^{k}$. More precisely, there are isomorphisms $T_{1, k}\left(B, R^{k *} \otimes R^{k}\right) \leftrightarrow T_{k}^{1}(B)$ commuting with the morphisms of the categories and taking the first corresponding connector into the second one. In this way one can entirely identify the pairs $\left(C_{1, k}\left(., R^{k *} \otimes R^{k}\right)\right.$, $\left.T_{1, k}\left(., R^{k *} \otimes R^{k}\right)\right)$ and $\left(C_{0}^{1}\left(R^{k},.\right), T_{k}^{1}\right)$. Moreover lemma 3.5 shows that both these categories are complete subcategories of $\mathscr{E}_{L}$. This means especially that each $\left(\mathscr{E}_{L}, T_{k}^{1}\right)$-connection is authomatically a $\left(C_{0}^{1}\left(R^{k},.\right), T_{k}^{1}\right)$-connection and each $\left(\mathscr{E}_{L}\right.$, $\left.T_{1, k}\left(., R^{k *} \otimes R^{k}\right)\right)$-connection is authomatically a $\left(C_{1, k}\left(., R^{k *} \otimes R^{k}\right), T_{1, k}\left(., R^{k *} \otimes\right.\right.$ $\left.\otimes R^{k}\right)$ )-connection and according to theorem 3.1 both they are in a one-to-one correspondence with the usual first order connections.

Returning to the general case let $\mathscr{T}$ be a connector as above. If $B_{1}, B_{2}$ are differentiable manifolds, there is a canonical morphism in $\mathscr{E}$

$$
\begin{gathered}
\left(\mathrm{id}_{B_{1} \times B_{2}}, \mathscr{T}\left(\pi_{1}\right) \times \mathscr{T}\left(\pi_{2}\right)\right):\left(B_{1} \times B_{2}, \mathscr{T}\left(B_{1} \times B_{2}\right)\right) \rightarrow \\
\rightarrow\left(B_{1} \times B_{2}, \mathscr{T}\left(B_{1}\right) \times \mathscr{T}\left(B_{2}\right)\right) .
\end{gathered}
$$

If $\mathscr{T}$ is local, this morphism is onto (lemma 3.3). We denoted here as usual by $\pi_{\mathbf{1}}$ or $\pi_{2}$ the canonical projection of the cartesian product onto its first or second component respectively. On the other hand if $x \in B_{1}$ is a fixed point, denote by $j^{x}: B_{2} \rightarrow B_{1} \times B_{2}$ the canonical injection taking $\eta$ into $(x, \eta)$ and analogously define $j^{y}: B_{1} \rightarrow B_{1} \times B_{2}$ for a fixed $y \in B_{2}$.

Definition 3.4. A connector $\mathscr{T}$ is called regular if for any two differentiable manifolds $B_{1}$ and $B_{2}$ the morphism (3.4) is one-to-one. A connector $\mathscr{T}$ is called semiregular if for any two differentiable manifolds $B_{1}$ and $B_{2}$ there exists a morphism $J_{1,2}$ : $: \mathscr{T}\left(B_{1}\right) \times \mathscr{T}\left(B_{2}\right) \rightarrow \mathscr{T}\left(B_{1} \times B_{2}\right)$ such that $\left(\mathscr{T}\left(\pi_{1}\right) \times \mathscr{T}\left(\pi_{2}\right)\right) J_{1,2}=\operatorname{id}_{\mathscr{T}\left(B_{1}\right) \times \mathscr{T}\left(B_{2}\right)}$ and

$$
\mathscr{T}\left(j^{x}\right) Y=J_{1,2}(\iota x, Y) ; \quad \mathscr{T}\left(j^{\nu}\right) X=J_{1,2}(X, \iota y)
$$

for each $x \in B_{1}, y \in B_{2}, X \in \mathscr{T}\left(B_{1}\right)$ and $Y \in \mathscr{T}\left(B_{2}\right)$. 
Note that each regular connector is semi-regular since $\pi_{i}\left(\mathscr{T}\left(\pi_{1}\right) \times \mathscr{T}\left(\pi_{2}\right)\right)$. . $\mathscr{T}\left(j^{x}\right) Y=\mathscr{T}\left(\pi_{i}\right) \mathscr{T}\left(j^{x}\right) Y=\mathscr{T}\left(\pi_{i} j^{x}\right) Y$ and this is equal to $\mathscr{T}([x]) Y=\iota X$ if $i=1$ and to $Y$ if $i=2$.

Lemma 3.6. If the connector $\mathscr{T}$ is onto $\mathscr{E}_{L}$ then it is semi-regular if $(x, Y) \rightarrow$ $\rightarrow \mathscr{T}\left(j^{x}\right) Y$ and $(X, y) \rightarrow \mathscr{T}\left(j^{y}\right) X$ are differentiable mappings from $B_{1} \times \mathscr{T}\left(B_{2}\right)$ and $\mathscr{T}\left(B_{1}\right) \times B_{2}$ respectively into $\mathscr{T}\left(B_{1} \times B_{2}\right)$.

Proof. First note that in this case (3.4) defines a linear mapping from $\mathscr{T}\left(B_{1} \times B_{2}\right)$ into $\mathscr{T}\left(B_{1}\right) \oplus \mathscr{T}\left(B_{2}\right)$. Now it suffices to put $J_{1,2}(X+Y)=\mathscr{T}\left(j^{P Y}\right) X+\mathscr{T}\left(j^{p X}\right) Y$.

Especially the connectors $\widetilde{T}_{r, k}(., A), \bar{T}_{r, k}(., A)$ and $T_{r, k}(., A)$ are semi-regular. We shall show recurrently that each connector $\tilde{J}_{s}^{r}\left(V_{p},.\right)$ is regular. Thus suppose that $\left(J_{1,2}\right)_{x}^{r-1}: \tilde{J}_{x}^{r-1}\left(V_{p}, B_{1}\right) \times \tilde{J}_{x}^{r-1}\left(V_{p}, B_{2}\right) \rightarrow \tilde{J}_{x}^{r-1}\left(V_{p}, B_{1} \times B_{2}\right)$ is defined for all $x$ in a neighbourhood of $s$ and satisfies $\left(J_{1,2}\right)_{x}^{r-1}\left(j^{r-1} \pi_{1} Z, j^{r-1} \pi_{2} Z\right)=Z$ for each $Z \in$ $\in \tilde{J}_{x}^{r-1}\left(V_{p}, B_{1} \times B_{2}\right)$ and $j^{r-1} \pi_{1}\left(J_{1,2}\right)_{x}^{r-1}(X, Y)=X, j^{r-1} \pi_{2}\left(J_{1,2}\right)_{x}^{r-1}(X, Y)=Y$ for each $X \in \tilde{J}_{x}^{r-1}\left(V_{p}, B_{1}\right)$ and $Y \in \tilde{J}_{x}^{r-1}\left(V_{p}, B_{2}\right)$. If we now put $\left(J_{1,2}\right)_{s}^{r}(X, Y)=j_{s}^{1}(x \rightarrow$ $\left.\rightarrow\left(J_{1,2}\right)_{x}^{r-1}(\varrho, \sigma)\right)$ where $X=j_{s}^{1} \varrho$ and $Y=j_{s}^{1} \sigma$, we get easily the required relation also for $\left(J_{1,2}\right)_{s}^{r}$. Thus we have established the regularity of $\tilde{J}_{s}^{r}\left(V_{p},.\right)$ and de facto also of $\bar{J}_{s}^{r}\left(V_{p},.\right)$ and $J_{s}^{r}\left(V_{p},.\right)$.

In general the connectors $\widetilde{T}_{r, k}(., A)$ need not be regular. Namely the relation $J_{1,2}\left(\mathscr{T}\left(\pi_{1}\right) Z+\mathscr{T}\left(\pi_{2}\right) Z\right)=Z$ is now of the form $\left\langle Z, \zeta j^{r}\left(j^{y}\right) j^{r} \pi_{1}\right\rangle+$ $+\left\langle Z, \zeta j^{r}\left(j^{x}\right) j^{r} \pi_{2}\right\rangle=\langle Z, \zeta\rangle$ for each $\zeta \in \widetilde{T}_{k}^{r *}\left(B_{1} \times B_{2}\right)_{(x, y)}, p Z=(x, y)$. But it can be seen that even for holonomic $\zeta$ the expression $\zeta j^{r}($ id $\times[y])+\zeta j^{r}([x] \times$ id $)$ need not be equal to $\zeta$ unless $r=1$. In fact if $r=1$ we have $\zeta=j_{(x, y)}^{1} \sigma$ and $j^{1}(\sigma($ id $\times$ $\times[y])+j^{1}(\sigma(x] \times$ id $\left.)\right)$ is equal to the 1-jet at $(x, y)$ of $(u, v) \rightarrow \sigma(u, y)+\sigma(x, v)$ which is the same as that of $(u, v) \rightarrow \sigma(u, v)$. This mens that $\widetilde{T}_{r, k}(., A)$ is regular iff $r=1$. One can also see that there is a commutative diagram

$$
\begin{gathered}
T_{k}^{1}\left(B_{1}\right) \times T_{k}^{1}\left(B_{2}\right) \longleftrightarrow T_{1, k}\left(B_{1}, R^{k *} \otimes R^{k}\right) \times T_{1, k}\left(B_{2}, R^{k *} \otimes R^{k}\right) \\
\uparrow \\
\downarrow \\
T_{k}^{1}\left(B_{1} \times B_{2}\right) \longleftrightarrow T_{1, k}\left(B_{1} \times B_{2}, R^{k *} \otimes R^{k}\right)
\end{gathered}
$$

where the correspondences in the rows are given by the natural isomorphisms between $T_{k}^{1}$ and $T_{1, k}\left(., R^{k *} \otimes R^{k}\right)$, and those in columns are given by the regularity of the corresponding connectors.

Let now again $\mathscr{T}$ be a general connector and suppose it is semi-regular. We shall define the prolongation of a composition law as follows. Let the composition $\varphi: M \subset B_{1} \times B_{2} \rightarrow B_{2}$ be given by $\varphi\left(z, z^{\prime}\right)=z \cdot z^{\prime}$, where $B_{1}, B_{2}, M$ are differentiable manifolds, $M$ being injected and regularly imbedded into $B_{1} \times B_{2}$ and $\varphi$ is differentiable. Suppose now $Z \in \mathscr{T}\left(B_{1}\right), Z^{\prime} \in \mathscr{T}\left(B_{2}\right), J_{1,2}\left(Z, Z^{\prime}\right) \in \mathscr{T}(M)$ and put

$$
Z \cdot Z^{\prime}=\mathscr{T}(\varphi) J_{1,2}\left(Z, Z^{\prime}\right) \in \mathscr{T}\left(B_{2}\right) .
$$


We have $p\left(Z . Z^{\prime}\right)=\varphi p J_{1,2}\left(Z, Z^{\prime}\right)=\varphi\left(p Z, p Z^{\prime}\right)=p Z \cdot p Z^{\prime}$ and $\iota z . \iota Z^{\prime}=\mathscr{T}(\varphi)$. $. \imath\left(z, z^{\prime}\right)=\imath\left(z \cdot z^{\prime}\right)$. In the case of three components this composition is associative if the underlying composition is associative.

Suppose now that the semi-regular connector $\mathscr{T}$ is also local and let $\Phi^{\bullet}$ be a differentiable groupoid, $a$ and $b$ as usual being its right and left unit projections respectively. Applying the above procedure we get in this way a differentiable groupoid structure in $\mathscr{T}(\Phi)$, the units being, by means of lemma 3.3, exactly all the elements of $\mathscr{T}(B) \subset \mathscr{T}(\Phi)$, where $B$ is the manifold of all units in $\Phi^{\circ}$. The composition $Z$. $Z^{\prime}$ of two elements $Z, Z^{\prime} \in \mathscr{T}(\Phi)$ is defined iff $\mathscr{T}(a) Z=\mathscr{T}(b) Z^{\prime}$. In fact if we take in lemma $3.1 B_{1}=M, B_{2}=\Phi \times \Phi, B_{2}^{\prime}=B, f=a \pi_{1}$ and $f^{\prime}=b \pi_{2}$, we have $Y \in$ $\in \mathscr{T}(M) \Leftrightarrow \mathscr{T}\left(a \pi_{1}\right) Y=\mathscr{T}\left(b \pi_{2}\right) Y$ and thus $J_{1,2}\left(Z, Z^{\prime}\right) \in \mathscr{T}(M) \Leftrightarrow \mathscr{T}(a) \mathscr{T}\left(\pi_{1}\right)$. . $J_{1,2}\left(Z, Z^{\prime}\right)=\mathscr{T}(b) \mathscr{T}\left(\pi_{2}\right) J_{1,2}\left(Z, Z^{\prime}\right)$, i.e. $\mathscr{T}(a) Z=\mathscr{T}(b) Z^{\prime}$. The inverse of $Z$ is $\mathscr{T}(\sigma) Z$, where $\sigma: \theta \rightarrow \theta^{-1}$ in $\Phi^{\bullet}$. Further from $b\left(z \cdot z^{\prime}\right)=b z$, i.e. $b \varphi\left(z, z^{\prime}\right)=$ $=b \pi_{1}\left(z, z^{\prime}\right)$ we get for $\mathscr{T}(a) Z=\mathscr{T}(b) Z^{\prime}$ the relation $\mathscr{T}(b)\left(Z . Z^{\prime}\right)=\mathscr{T}\left(b \pi_{1}\right)$. . $J_{1,2}\left(Z . Z^{\prime}\right)=\mathscr{T}(b) Z$ and analogously $\mathscr{T}(a)\left(Z . Z^{\prime}\right)=\mathscr{T}(a) Z^{\prime}$. From these relations we obtain now easily all the axioms of the differentiable groupoid $\mathscr{T}(\Phi)^{\bullet}$ with the right and left unit projections $\mathscr{T}(a)$ and $\mathscr{T}(b)$ respectively. Note that if $\mathscr{T}$ is into $\mathscr{E}_{L}$ then the above composition in $\mathscr{T}(\Phi)^{\bullet}$ defines a linear mapping of a subbundle of $\mathscr{T}(\Phi) \otimes \mathscr{T}(\Phi)$ into $\mathscr{T}(\Phi)$.

If the differentiable groupoid $\Phi^{\bullet}$ is a differentiable groupoid of operators on the manifold $E$ then $\mathscr{T}(\Phi)$ is a differentiable groupoid of operators on the manifold $\mathscr{T}(E)$, the composition $Z . X, Z \in \mathscr{T}(\Phi), X \in \mathscr{T}(E)$ being defined iff $\mathscr{T}(\bar{p}) X=\mathscr{T}(a) Z$, where $\bar{p}: E \rightarrow B$ is the natural projection. Suppose now that $\Phi^{\bullet}$ is locally trivial, i.e. let $\{U\}$ be an open covering of $B$ such that for each such $U$ there exists a differentiable mapping $\theta_{U}: U \rightarrow \Phi$ with $a \theta_{U}=c, b \theta_{U}=\mathrm{id}_{U}$. For each $U$ we define now $\Theta_{U}$ : $: \mathscr{T}(U) \rightarrow \mathscr{T}(\Phi)$ as $\Theta_{U}=\mathscr{T}\left(\theta_{U}\right)$. We have $\mathscr{T}(a) \Theta_{U}=\mathscr{T}\left(a \theta_{U}\right)=\mathscr{T}([c])=\imath c$ and $\mathscr{T}(b) \Theta_{U}=\mathscr{T}\left(\operatorname{id}_{U}\right)=\operatorname{id}_{\mathscr{T}(U)}$. Thus also $\mathscr{T}(\Phi)^{\bullet}$ is locally trivial and if $E=E(B, F$, $\left.G^{*}, H, \Phi^{*}\right)$ then analogous considerations as in the first chapter lead to the conclusion that $\mathscr{T}(E)$ is a fibre bundle

$$
\mathscr{T}(E)\left(\mathscr{T}(B), \mathscr{T}(\bar{p})^{-1} \iota c, \mathscr{T}(G)^{\bullet}, \mathscr{T}(H), \mathscr{T}(\Phi)^{\bullet}\right) .
$$

Hereby $\mathscr{T}(H) \subset \mathscr{T}(\Phi)$ is a principal fibre bundle characterized by the equation $\mathscr{T}(a) Z=\imath c$ and $\mathscr{T}(G)^{\bullet} \subset \mathscr{T}(\Phi)^{\bullet}$ is a Lie group characterized by the equations $\mathscr{T}(a) Z=\mathscr{T}(b) Z=\iota c$. On the other hand if $g \in G$ is fixed, we have $g: H \rightarrow H$ and thus $\mathscr{T}(g): \mathscr{T}(H) \rightarrow \mathscr{T}(H)$.

Lemma 3.7. If the local connector $\mathscr{T}$ is semi-regular then for each $g \in G$ and $Z \in \mathscr{T}(H)$ we have

$$
\mathscr{T}(g) Z=Z . \imath g
$$

Proof. From $g: z \rightarrow \varphi j^{g} z$ we get $\mathscr{T}(g) Z=\mathscr{T}(\varphi) \mathscr{T}\left(j^{g}\right) Z$ which by means of (3.5) and (3.7) is equal to $\mathscr{T}(\varphi) J_{1,2}(Z, \imath g)=Z . \imath g$. 
Note that if $\mathscr{T}=T_{1}^{1}$ then the operation given by (3.9) corresponds to the classical one denoted e.g. in [14] by $R_{g}$. Analogously if $\bar{h} \in H^{-1}$ then $\mathscr{T}(\bar{h}) Z=Z$. $\iota \bar{h}$ for each $Z \in \mathscr{T}(H)$.

Denote by $\mathscr{T}_{e}(G)$ the subset of all elements $Z_{0}$ in $\mathscr{T}(G)$ for which $p Z_{0}=e=\tilde{c}$. Each element $Z \in \mathscr{T}(G)$ can be uniquely written as $Z=Z_{0} . \iota g$, where $g \in G$ and $Z_{0} \in \mathscr{T}_{e}(G)$. In fact it suffices to take $Z_{0}=Z$. $\iota \sigma p Z$ and $g=p Z$.

Lemma 3.8. If the local semi-regular connector $\mathscr{T}$ is into $\mathscr{E}_{L}$ then for each $Z_{0}, Z_{0}^{\prime} \in$ $\in \mathscr{T}_{e}(G)$ we have $Z_{0} \cdot Z_{0}^{\prime}=Z_{0}+Z_{0}^{\prime}$.

Proof. From lemma 3.6 we have $Z_{0} \cdot Z_{0}^{\prime}=\mathscr{T}(\varphi)\left(\mathscr{T}\left(j^{e}\right) Z_{0}+\mathscr{T}\left(j^{e}\right) Z_{0}^{\prime}\right)=$ $=Z_{0} \cdot \iota e+\iota e \cdot Z_{0}^{\prime}$.

Corollary. If the local semi-regular connector $\mathscr{T}$ is into $\mathscr{E}_{L}$ and $Z_{0} \in \mathscr{T}_{e}(G)$, $X \in \mathscr{T}(H)$ then $X . Z_{0}=X+\iota p X . Z_{0}$.

Note again that if $\mathscr{T}=T_{1}^{1}, \mathscr{T}_{e}(G)$ is the Lie algebra of $G$ and $h \rightarrow \iota h . Z_{0}$ is the fundamental vector field $Z_{0}^{*}$ corresponding to $Z_{0} \in \mathscr{T}_{e}(G)$ (cf. [14]).

We can now define the infinitesimal $\mathscr{T}$-displacements of the fibre $E_{\boldsymbol{x}}$ as elements of $\mathscr{T}(\Phi)_{x}^{+}$, where $\mathscr{T}(\Phi)^{+}=\{Z \in \mathscr{T}(\Phi) \mid p Z \in B, \mathscr{T}(a) Z=\imath p Z\}$. Denote further by $\mathscr{T}(H) / G^{\bullet}$ the set of all right cosets with respect to the action of $G^{\bullet}$ upon $\mathscr{T}(H)$ endowed with the natural coset differentiable structure. If we now denote

$$
\begin{array}{ll}
\tilde{\pi} \quad: Z \rightarrow Z \cdot \iota \sigma p Z ; \\
\pi_{\tilde{G}}: Z \rightarrow Z \cdot \iota G ; \\
\mathscr{J}^{-1}: Z . \imath G \rightarrow Z . \iota \sigma p Z \text { its inverse being } \\
\mathscr{J}^{-1}: Z \rightarrow Z . \iota \theta, \text { where } \theta \in H \text { is any such that } b \theta=p Z,
\end{array}
$$

we get a commutative diagram

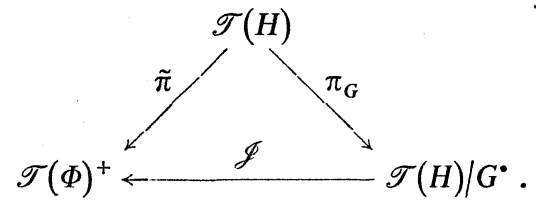

We have $(\mathscr{T}(B), \mathscr{T}(H)) \in \mathscr{E},\left(\mathscr{T}(B), \mathscr{T}(\Phi)^{+}\right) \in \mathscr{E},\left(\mathscr{T}(B), \mathscr{T}(H) / G^{*}\right) \in \mathscr{E},(B, \mathscr{T}(H)) \in$ $\in \mathscr{E},\left(\mathrm{id}_{B}, \pi_{\mathrm{G}}\right) \in \mathscr{E},\left(\mathrm{id}_{B}, \tilde{\pi}\right) \in \mathscr{E}$ and also

$$
\left(B, \mathscr{T}(\Phi)^{+}\right) \in \mathscr{E}, \quad\left(B, \mathscr{T}(H) / G^{*}\right) \in \mathscr{E}, \quad(\sim, \tilde{J}) \in \mathscr{E}, \quad\left(\mathrm{id}_{B}, \mathscr{J}\right) \in \mathscr{E},
$$

where $\tilde{\jmath}: \mathscr{T}(\Phi)^{+} \rightarrow \mathscr{T}(\Phi)$ is the natural injection. It is not difficult to see that if $\mathscr{T}$ is into $\mathscr{E}_{L}$ then in (3.10) the category $\mathscr{E}$ can be replaced by $\mathscr{E}_{L}$.

Theorem 3.2. Let $\mathscr{T}$ be a local semi-regular connector. Then each $(\mathscr{V}, \mathscr{T})-$ 
connection $\mathscr{X}$ on a fibre bundle $E\left(B, F, G^{\bullet}, H, \Phi^{\bullet}\right)$ can be uniquely characterized by a differentiable section $\mathscr{Y}$ in the fibred manifold $\left(\mathscr{T}(B), \mathscr{T}(\Phi)^{+}\right)$satisfying

$$
(\sim, \tilde{\jmath} \mathscr{Y}) \in \mathscr{V},
$$

or by a differentiable section $\mathscr{Z}$ in the fibred manifold $\left(\mathscr{T}(B), \mathscr{T}(H) / G^{\bullet}\right)$ satisfying

$$
(\sim, \tilde{J} \mathscr{J} \mathscr{Z}) \in \mathscr{V}
$$

The theorem is an immediate consequence of our previous discussion. Note that if $\mathscr{V}=\mathscr{E}$ then the conditions (3.11) and (3.12) are automatically fulfilled and if $\mathscr{V}=\mathscr{E}_{L}$ they can be replaced by $\left(\mathrm{id}_{B}, \mathscr{Y}\right) \in \mathscr{E}_{L}$ and $\left(\mathrm{id}_{B}, \mathscr{Z}\right) \in \mathscr{E}_{L}$ respectively. Note that in the case $\mathscr{V}=\mathscr{E}_{L}$ and $\mathscr{T}=\bar{T}_{q, 1}$ this theorem yields the definition of semiholonomic connections of order $q$ brought in [12], in general not equivalent with that of Ehresmann (cf. theorem 3.1).

Definition 3.5. A category $\mathscr{V} \subset \mathscr{E}$ is called local if it has the following properties:

(1) If $(f, F):\left(B_{1}, C_{1}\right) \rightarrow\left(B_{2}, C_{2}\right)$ belongs to $\mathscr{V}$ and $U \subset B_{1}$ is open, then also the restriction $\left(\left.f\right|_{U},\left.F\right|_{p^{-1}(U)}\right):\left(U, p^{-1}(U)\right) \rightarrow\left(W, p^{-1}(W)\right)$, where $f(U) \subset W \subset B_{2}$ and $W$ is open, belongs to $\mathscr{V}$.

(2) If $\{U\}$ is an open covering of the differentiable manifold $B_{1},\left(B_{1}, C_{1}\right)$ and $\left(B_{2}, C_{2}\right)$ belong to $\mathscr{V}$ and if in each $U$ there is a morphism $\left(f_{U}, F_{U}\right)$ : $:\left(U, p^{-1}(U)\right) \rightarrow\left(B_{2}, C_{2}\right)$ from $\mathscr{V}$ such that these morphisms coincide in the intersection of their domains, then $(f, F):\left(B_{1}, C_{1}\right) \rightarrow\left(B_{2}, C_{2}\right)$, obtained from them by "superposition", also belongs to $\mathscr{V}$.

Note that the categories $\mathscr{E}, \mathscr{E}^{0}, \mathscr{E}_{L}, \widetilde{C}_{s}^{r}\left(V_{p},.\right), \bar{C}_{s}^{r}\left(V_{p},.\right), C_{s}^{r}\left(V_{p},.\right), \widetilde{C}_{r, k}(., A)$, $\bar{C}_{r, k}(., A)$ and $C_{r, k}(., A)$ are all local. In general if $\mathscr{V}$ is isomorphic with $\tilde{J}^{r}$ or $\bar{J}^{r}$ or $\boldsymbol{J}^{r}$, then it is local.

Theorem 3.3. Let $\mathscr{T}$ be a local semi-regular connector into a local category $\mathscr{V}$. Then each $(\mathscr{V}, \mathscr{T})$-connection $\mathscr{X}$ on a fibre bundle $E\left(B, F, G^{*}, H, \Phi^{*}\right)$ can be uniquely characterized by prescribing for each $h \in H$ a subspace $\mathscr{T}(H)_{h}^{+}$of $\mathscr{T}(H)_{h}$ satisfying

(1) $\mathscr{T}(b) \mathscr{T}(H)_{h}^{+}=\mathscr{T}(B)_{b h}$;

(2) $\mathscr{T}(H)_{h g}^{+}=\mathscr{T}(H)_{h}^{+} . \iota g$ for each $g \in G$;

(3) there exists an open covering $\{U\}$ of $B$ such that for each $U$ there is an injective morphism $\left(\xi_{U}, \Xi_{U}\right):(U, \mathscr{T}(U)) \rightarrow(H, \mathscr{T}(H))$ belonging to $\mathscr{V}$ and satisfying $b \xi_{U}=\mathrm{id}_{U}$ and $\Xi_{U}\left(\mathscr{T}(B)_{x}\right)=\mathscr{T}(H)_{\xi U(x)}^{+}$.

Proof. Let $\mathscr{X}$ be a $(\mathscr{V}, \mathscr{T})$-connection on $E$. Let $U$ be any fixed open subset of $B$ given by the local triviality of $\Phi^{*}$, i.e. $u \rightarrow \theta_{u}$ is a differentiable mapping $U \rightarrow \Phi$ with $a \theta_{u}=c$ and $b \theta_{u}=u$. Denote by $\mathscr{X}_{U}$ the restriction of $\mathscr{X}$ to $\mathscr{T}(U) \subset \mathscr{T}(B)$. Since $\mathscr{V}$ is local, $\left(\sim, \mathscr{X}_{U}\right):(U, \mathscr{T}(U)) \rightarrow\left(a^{-1}(U) \cap b^{-1}(U), \mathscr{T}\left(a^{-1}(U) \cap b^{-1}(U)\right)\right) \subset$ 
$\subset(\Phi, \mathscr{T}(\Phi))$ belongs to $\mathscr{V}$ as well as $\left(f_{U}, \mathscr{T}\left(f_{U}\right)\right):\left(a^{-1}(U) \cap b^{-1}(U), \mathscr{T}\left(a^{-1}(U) \cap\right.\right.$ $\left.\left.\cap b^{-1}(U)\right)\right) \rightarrow(H, \mathscr{T}(H))$ given by $f_{U}(z)=z \cdot \theta_{a z}$. Thus also $\left(\xi_{U}, \Xi_{U}\right)=\left(f_{U}, \mathscr{T}\left(f_{U}\right)\right)$. . $\left(\sim, \mathscr{X}_{U}\right)$ belongs to $\mathscr{V}$. We have the formula

$$
\Xi_{U} Z=\mathscr{X}_{U} Z \cdot \iota \theta_{p Z} .
$$

In fact, denote by $\Phi_{z}=\left\{y \in a^{-1}(U) \cap b^{-1}(U) \mid a y=z \in U\right\}$ and let $f_{z}=\left.f_{U}\right|_{\Phi_{z}}$. Analogously as in lemma 3.7 we have $\mathscr{T}\left(f_{z}\right) Y=Y . \iota \theta_{z}$ for all $Y \in \mathscr{T}\left(\Phi_{z}\right)$ and since $\mathscr{T}(a) \mathscr{X}_{U} Z=\iota p Z$ we get the relation (3.13) for each $Z \in \mathscr{T}(U)$. From there we can easily see that $\mathscr{T}(H)_{h}^{+}=\Xi_{U}\left(\mathscr{T}(B)_{b h}\right) \cdot \iota g$, where $h=\xi_{U}(b h) \cdot g$ is defined independently of the choice of $U$ containing $b h$. We have also $\mathscr{T}(b) \Xi_{U} Z=\mathscr{T}(b) \mathscr{X} Z=Z$.

Conversely suppose there are given subspaces as in the theorem and define the connection $\mathscr{X}$ by

$$
\mathscr{X} Z=\tilde{\jmath} \tilde{\pi} \Xi_{U}^{*} Z, \quad p Z \in U,
$$

where $\Xi_{U}^{*}=\Xi_{U} \Sigma_{U}^{-1}$ and $\Sigma_{U}=\mathscr{T}(b) \Xi_{U}: \mathscr{T}(U) \rightarrow \mathscr{T}(U)$ is one-to-one. In fact, since $\Xi_{U}$ is injective this follows from (1). Note that $\left(\xi_{U}, \Xi_{U}^{*}\right) \in \mathscr{V}$.

Next we are going to show that $\tilde{\pi} \Xi_{U}^{*} Z$ does not depend on the choice of $U$. Thus let $p Z=z \in U \cap U^{\prime}$. If $\xi_{U^{\prime}}(z)=\xi_{U}(z) \cdot \gamma$ for some $\gamma \in G$, we have $\Xi_{U^{\prime}}\left(\mathscr{T}(B)_{z}\right)=$ $=\Xi_{U}\left(\mathscr{T}(B)_{z}\right) \cdot \iota \gamma$ and thus also $\Xi_{U}^{*}\left(\mathscr{T}(B)_{z}\right)=\Xi_{U}^{*}\left(\mathscr{T}(B)_{z}\right) \cdot \iota \gamma$. This means that for each $Z \in \mathscr{T}(B)_{z}$ one finds $F(Z)$ such that $\Xi_{U^{\prime}}^{*}(F(Z))=\Xi_{U}^{*} Z \cdot \iota \gamma$. But since $\mathscr{T}(b) \Xi_{U^{\prime}}^{*}=$ $=\mathscr{T}(b) \Xi_{U}^{*}=\operatorname{id}_{\mathscr{F}(U)}$ we get $F(Z)=Z$ and thus $\Xi_{U}^{*}, Z=\Xi_{U}^{*} Z . \iota \gamma$ which implies $\tilde{\pi} \Xi_{U}^{*}, Z=\tilde{\pi} \Xi_{U}^{*} Z$. Note that we have also

$$
\Xi_{U}^{*}\left(\mathscr{T}(B)_{z}\right) \cdot \iota g=\mathscr{T}(H)_{\xi(z) \cdot g}^{+} \cdot
$$

The relation $p \mathscr{X} Z=\widetilde{p Z}$ is evident, $\mathscr{T}(b) \mathscr{X Z}=\mathscr{T}(b) \Xi_{U}^{*} Z=Z$ and $\mathscr{T}(a) \mathscr{X} Z=$ $=\mathscr{T}(a)\left(\Xi_{U}^{*} Z . \iota \sigma p \Xi_{U}^{*} Z\right)=\iota a \sigma p \Xi_{U}^{*} Z=\iota p \mathscr{T}(b) \Xi_{U}^{*} Z=\iota p Z$. We first show that $(\sim b, \tilde{j} \tilde{\pi})$ belongs to $\mathscr{V}$. In fact, for each fixed $h \in H,\left(\sim b, \mathscr{T}\left(h^{-1}\right)\right) \in \mathscr{V}$ and $\left.\tilde{j} \tilde{\pi}\right|_{p^{-1}(h)}=$ $\mathscr{T}\left(h^{-1}\right)$ and thus according to the locality of $\mathscr{V}$ we have $(\sim b, \tilde{\mathrm{j}} \tilde{\pi}) \in \mathscr{V}$. Further for each fixed $U$ we have the decomposition $\left(\left.\sim\right|_{U},\left.\mathscr{X}\right|_{\mathscr{T}(U)}\right)=\left(\left.\sim b\right|_{b_{H^{-1}}(U)},\left.\tilde{\pi} \tilde{\pi}\right|_{\left.\mathscr{T}_{\left(b_{H^{-1}}(U)\right)}\right)}\right)$. . $\left(\xi_{U}, \Xi_{U}^{*}\right)$ and hence again from the locality of $\mathscr{V}$ we get $(\sim, \mathscr{X}) \in \mathscr{V}$ and this completes the proof.

If $\mathscr{T}=\widetilde{T}_{r, 1}$ and $\mathscr{V}=\widetilde{C}_{r, 1}(., R)$ then the assumptions of this theorem are those of the definition of a non-holonomic connection of order $r$ in the form given in [1] and [2]. If here $r=1$ then, as we have seen, one may replace $C_{1,1}(., R)$ by $\mathscr{E}_{L}$ without loss of generality and the $\mathscr{T}(H)_{h}^{+}$are exactly the horizontal linear subspaces of the tangent spaces to $H$ (cf. [14]).

Suppose again that $\mathscr{T}$ is a local semi-regular connector into $\mathscr{V}$. If $\mathscr{X}$ is a $(\mathscr{V}, \mathscr{T})$ connection on the fibre bundle $E(B, F, G \cdot H, \Phi \cdot)$ we call

$$
\nabla_{\mathscr{X}} Z=\mathscr{T}(\sigma) \mathscr{X} \mathscr{T}(\bar{p}) Z . Z
$$

the absolute differential of $Z$ with respect to $\mathscr{X}$. The morphism $\left(\mathrm{id}_{E}, \nabla_{\mathscr{X}}\right):(E, \mathscr{T}(E)) \rightarrow$ 
$\rightarrow(E, \mathscr{T}(E))$ belongs to $\mathscr{E}$ and if $\mathscr{V} \subset \mathscr{E}_{L}$ then it belongs even to $\mathscr{E}_{L}$. Note that the composition in (3.15) is defined since $\mathscr{T}(a) \mathscr{T}(\sigma) \mathscr{X} \mathscr{T}(\bar{p}) Z=\mathscr{T}(b) \mathscr{X} \mathscr{T}(\bar{p}) Z=$ $=\mathscr{T}(\bar{p}) Z$. Moreover we have, using the prolongation of $\bar{p}(\theta . z)=b \theta$, the relation $\mathscr{T}(\bar{p})(\mathscr{T}(\sigma) \mathscr{X} \mathscr{T}(\bar{p}) Z . Z)=\mathscr{T}(b) \mathscr{T}(\sigma) \mathscr{X} \mathscr{T}(\bar{p}) Z=\iota p \mathscr{T}(\bar{p}) Z=\iota \bar{p} p Z$, which means that

$$
\nabla_{\mathscr{X}} Z \in \mathscr{T}\left(E_{\bar{p} p Z}\right) .
$$

Analogous results are obtained if one replaces $E$ by $H$. If $Z \in \mathscr{T}(H)$ we define again a horizontal projection, called its absolute differential with respect to $\mathscr{X}$,

$$
\nabla_{\mathscr{X}} Z=\mathscr{T}(\sigma) \mathscr{X} \mathscr{T}(b) Z . Z \in \mathscr{T}\left(H_{b p Z}\right) .
$$

Define now for each $Z \in \mathscr{T}(H)$ the element

$$
\omega(Z)=\iota \sigma p Z \cdot \nabla_{\mathscr{X}} Z .
$$

Note that the composition in (3.18) is always defined since $\mathscr{T}(a) \iota \sigma p Z=\imath b p Z=$ $=\mathscr{T}(b) \nabla_{\mathscr{X}} Z$.

Theorem 3.4. Let $\mathscr{T}$ be a local semi-regular connector into a local category $\mathscr{V}$. Then each $(\mathscr{V}, \mathscr{T})$-connection $\mathscr{X}$ on a fibre bundle $E\left(B, F, G \cdot H, \Phi^{\bullet}\right)$ can be uniquely characterized by a differentiable mapping $\omega: \mathscr{T}(H) \rightarrow \mathscr{T}_{e}(G)$ which satisfies

(1) $\omega(Z . Y)=\iota \sigma p Y . \omega(Z) . Y$ for each $Z \in \mathscr{T}(H)$ and $Y \in \mathscr{T}(G)$;

(2) $(\sim b, \overline{\mathscr{X}}):(H, \mathscr{T}(H)) \rightarrow(\Phi, \mathscr{T}(\Phi))$ belongs to $\mathscr{V}$, where $\overline{\mathscr{X}} Z=Z . \mathscr{T}(\sigma) \omega(Z)$. . $\iota \sigma p Z$.

The correspondence between $\omega$ and $\mathscr{X}$ is given by (3.18).

Proof. Let $\mathscr{X}$ be a connection and let $\omega$ be given by (3.18). Since $\mathscr{T}(b) \omega(Z)=$ $=\mathscr{T}(b) \iota \sigma p Z=\iota a p Z=\iota c$ and $p \omega(Z)=\sigma p Z \cdot p \mathscr{T}(\sigma) \mathscr{X} \mathscr{T}(b) Z \cdot p Z=\sigma p Z . \sigma \sim$ $\sim p \mathscr{T}(b) Z \cdot p Z=\sim a p Z=e$ we have really $\omega: \mathscr{T}(H) \rightarrow \mathscr{T}_{e}(G)$. Further $\omega(Z, Y)=$ $=\imath(\sigma p Y . \sigma p Z) . \mathscr{T}(\sigma) \mathscr{X} \mathscr{T}(b) Z . Z . Y$ and this gives (1). Analogously (2), since $\overline{\mathscr{X}} Z=\mathscr{X} \mathscr{T}(b) Z$.

Conversely let $\omega$ be given as in the theorem and let $\{U\}$ be a covering of $B$ given by the local triviality of $\Phi^{\circ}$. Thus for each $U$ we have again a differentiable section $\theta_{U}: U \rightarrow H$ and define $\mathscr{X}_{U}=\overline{\mathscr{X}} \mathscr{T}\left(\theta_{U}\right): \mathscr{T}(U) \rightarrow \mathscr{T}(\Phi)$. We have clearly $\left(\sim, \mathscr{X}_{U}\right) \in$ $\in \mathscr{V}$ and if $Z \in \mathscr{T}(U) \cap \mathscr{T}\left(U^{\prime}\right)$ we get $\mathscr{X}_{U} Z=\mathscr{X}_{U^{\prime}} Z$. In fact, we have namely $\mathscr{T}(b) X=$ $=\mathscr{T}(b) X^{\prime}$, where $X=\mathscr{T}\left(\theta_{U}\right) Z$ and $X^{\prime}=\mathscr{T}\left(\theta_{U^{\prime}}\right) Z$ and this implies $Y=\mathscr{T}(\sigma) X$. .$X^{\prime} \in \mathscr{T}(G)$. Thus using $(1)$ one derives $\bar{X} X^{\prime}=\overline{\mathscr{X}}(X . Y)=X . Y . \mathscr{T}(\sigma)(\iota \sigma p Y$. $. \omega(X) . Y) \cdot \iota \sigma p X=X . Y . \mathscr{T}(\sigma) Y . \mathscr{T}(\sigma) \omega(X) \cdot \iota p Y . \iota \sigma p X=X . \mathscr{T}(\sigma) \omega(X)$. $. \iota \sigma p X=\overline{\mathscr{X}} X$. Therefore we can apply (2) of definition 3.5 and this gives $(\sim, \mathscr{X}) \in \mathscr{V}$. One verifies now easily the relations $\mathscr{T}(a) \mathscr{X}=\iota p$ and $\mathscr{T}(b) \mathscr{X}=\mathrm{id}_{\mathscr{T}(B)}$ and completes the proof. 
Note that if $\mathscr{V}=\mathscr{E}$ then (2) is satisfied automatically and if $\mathscr{V}=\mathscr{E}_{L}$ then (2) can be replaced by assuming $\omega$ to be linear (cf. theorem 3.5). In general (1) in theorem 3.4 induces $\omega(\iota h . Y)=\omega(\iota h) . Y$ for each $h \in H$ and $Y \in \mathscr{T}_{e}(G)$ and $\omega(Z . \iota g)=$ $=\operatorname{Ad}\left(g^{-1}\right) \omega(Z)$, where $Z \in \mathscr{T}(H), g \in G$ and $\operatorname{Ad}\left(g^{-1}\right) Y=\iota g^{-1} . Y . \imath g$.

Lemma 3.9. Let $\mathscr{T}$ be a local semi-regular connector into $\mathscr{E}_{L}$ and let $\omega: \mathscr{T}(H) \rightarrow$ $\rightarrow \mathscr{T}_{e}(G)$ be linear and satisfy

$$
\begin{aligned}
& \omega(\iota h . Y)=Y \text { for each } h \in H \text { and } Y \in \mathscr{T}_{e}(G), \\
& \omega(Z . \iota g)=\operatorname{Ad}\left(g^{-1}\right) \omega(Z) \text { for each } Z \in \mathscr{T}(H) \text { and } g \in G .
\end{aligned}
$$

Then $\omega$ satisfies (1) of the theorem 3.4 .

Proof. We have for $Y \in \mathscr{T}(G)$ denoting $g=p Y$ and $Y_{0}=Y . \iota \sigma p Y$ the relation $\omega(Z . Y)=\omega\left(Z . Y_{0} . \iota g\right)$ which equals by (3.20) and the corollary of lemma 3.8 to $\operatorname{Ad}\left(g^{-1}\right)\left(\omega(Z)+\omega\left(\iota p Z . Y_{0}\right)\right)$ and this is by means of (3.19) and lemma 3.8 equal to $\operatorname{Ad}\left(g^{-1}\right)\left(\omega(Z) . Y_{0}\right)$ which is the required result $\iota \sigma p Y . \omega(Z) . Y_{0} . \iota p Y$.

If $\mathscr{T}$ is into $\mathscr{E}_{L}$ and $\omega$ is linear then necessarily $\omega(\iota h)=\iota e$ for each $h \in H$. The following lemma shows the relation between theorem 3.3 and theorem 3.4 .

Lemma 3.10. Let $\mathscr{T}$ be a local semi-regular connector into a local $\mathscr{V}$. If $\mathscr{X}$ is $a(\mathscr{V}, \mathscr{T})$-connection on a fibre bundle $E$ then $\mathscr{T}(H)^{+}$given by theorem 3.3 and $\omega$ given by theorem 3.4 satisfy

$$
Z \in \mathscr{T}(H)^{+} \Leftrightarrow \omega(Z)=\iota e .
$$

Proof. With the notations in the proof of theorem 3.3 we have by means of (3.13) $Z \in \mathscr{T}(H)_{h}^{+} \Leftrightarrow Z=\mathscr{X} V . \iota \theta_{p V}$ for some $V \in \mathscr{T}(B)_{b h}$ and consequently $\omega(Z)=$ $=\iota \sigma\left(p \mathscr{X} V . \theta_{p V}\right) . \mathscr{T}(\sigma) \mathscr{X} \mathscr{T}(b) \mathscr{X} V . \mathscr{X} V . \iota \theta_{p V}=\imath \sigma \theta_{p V} \cdot \mathscr{T}(\sigma) \mathscr{X} V . \mathscr{X} V . \iota \theta_{p V}=\imath \sim$ $\sim a \theta_{p V}=\imath e$. Conversely if $\omega(Z)=\iota e$ then $\nabla_{\mathscr{X}} Z=\iota p Z$, i.e. $Z=\mathscr{X} \mathscr{T}(b) Z \cdot \iota p Z$ and there exists in a neighbourhood of $b p Z$ a local section $x \rightarrow \theta_{x}$ such that $a \theta_{x}=c$, $b \theta_{x}=x$ and $\theta_{b p Z}=p Z$. Thus $Z \in \mathscr{T}(H)_{p Z}^{+}$.

Definition 3.6. A category $\mathscr{V} \subset \mathscr{E}$ is called fine if it has the following property:

Let $(f, F):\left(B_{1}, C_{1}\right) \rightarrow\left(B_{2}, C_{2}\right)$ belong to $\mathscr{E}$ and let for each $z \in B_{1}$ there exist $\left(f_{z}, F_{z}\right):\left(B_{1}, C_{1}\right) \rightarrow\left(B_{2}, C_{2}\right)$ belonging to $\mathscr{V}$ such that $f_{z}(z)=f(z)$ and $\left.F_{z}\right|_{p^{-1}(z)}=$ $=\left.F\right|_{p^{-1}(z)}$. Then $(f, F)$ belongs to $\mathscr{V}$.

Note that all the categories $\mathscr{E}, \mathscr{E}_{L}, \widetilde{C}_{s}^{r}\left(V_{p},.\right), \bar{C}_{s}^{r}\left(V_{p},.\right), C_{s}^{r}\left(V_{p},.\right), \widetilde{C}_{r, k}(., A)$, $\bar{C}_{r, k}(., A), C_{r, k}(., A)$ are fine.

Theorem 3.5. Let $\mathscr{T}$ be a local semi-regular connector into a fine category $\mathscr{V} \subset \mathscr{E}_{L}$. Then each $(\mathscr{V}, \mathscr{T})$-connection $\mathscr{X}$ on a fibre bundle $E\left(B, F, G^{\bullet}, H, \Phi^{*}\right)$ can be uniquely 
characterized by a linear differentiable mapping $\omega: \mathscr{T}(H) \rightarrow \mathscr{T}_{e}(G)$ satisfying (3.19), (3.20) and

$$
\mathscr{V} \ni\left(\mathrm{id}_{H}, \iota p . \omega\right):(H, \mathscr{T}(H)) \rightarrow(H, \mathscr{T}(H)),
$$

where the correspondence between $\mathscr{X}$ and $\omega$ is given again by (3.18).

Proof. According to lemma 3.9 we need to show only that $(\sim, \mathscr{X}) \in \mathscr{V}$ implies (3.22) and that (3.22) implies (2) of theorem 3.4. Thus let $\mathscr{X}$ be a connection and since $\mathscr{T}$ is into $\mathscr{E}_{L}$ we can write $\left(\mathrm{id}_{H}, \iota p . \omega\right)=\left(\mathrm{id}_{H}, \nabla_{\mathscr{X}}\right)=(\varphi, \mathscr{T}(\varphi))(\sim b \times$ $\left.\times \mathrm{id}_{H}, \nabla_{1}+\nabla_{2}\right)$, where $\nabla_{1} Z=\mathscr{T}\left(j^{p Z}\right) \mathscr{T}(\sigma) \mathscr{X} \mathscr{T}(b) Z$ and $\nabla_{2} Z=\mathscr{T}\left(j^{\sim p Z}\right) Z$. But since $\mathscr{V}$ is fine and $\left(\sim b \times \mathrm{id}_{H}, \nabla_{1}\right) \in \mathscr{E}$ we conclude that it belongs to $\mathscr{V}$ whereby for each $h \in H$ we put $f_{h}(x)=(\sim b x, h)$ and $F_{h}(X)=\mathscr{T}\left(j^{h}\right) \mathscr{T}(\sigma) \mathscr{X} \mathscr{T}(b) X$. Analogously $\left(\sim b \times \mathrm{id}_{H}, \nabla_{2}\right) \in \mathscr{V}$ and hence also $\left(\mathrm{id}_{H}, \nabla_{\mathscr{X}}\right) \in \mathscr{V}$. Observing that on the other hand $\overline{\mathscr{X}} Z=Z . \mathscr{T}(\sigma)(\iota p Z . \omega(Z))$ we prove analogously the second implication and complete the proof.

This characterization of a connection coincides de facto in the case $\mathscr{T}=\widetilde{T}_{r, 1}$ and $\mathscr{V}=\tilde{C}_{r, 1}(., R)$ with the one given in [1] and [2], and if $\mathscr{V}=T_{1}^{1}$ it corresponds to the well known classical property (cf. [14]).

We finish the paper with the following definitions and theorem which lead to a generalization of the notion of contact elements in order to give another characterization of some $(\mathscr{V}, \mathscr{T})$-connections.

Definition 3.7. Let $\bar{\Gamma}$ be a semigroup with a single unit element, $\Gamma \subset \bar{\Gamma}$ the subgroup of all its inversible elements, and let there be given in $\bar{\Gamma}$ a differentiable structure compatible with the algebraic structure and inducing in $\Gamma$ a Lie group structure. We say that $\bar{\Gamma}$ is a semigroup of operators on a category $\mathscr{V} \subset \mathscr{E}$, or, briefly, that it operates on $\mathscr{V}$ if there is a differentiable mapping $C \times \bar{\Gamma} \rightarrow C$ denoted by $(X, \gamma) \rightarrow$ $\rightarrow X \gamma$ for each object $(B, C) \in \mathscr{V}$ satisfying

$$
p(X \gamma)=p X \text { for each } X \in C \text { and } \gamma \in \bar{\Gamma} \text {; }
$$

(2) $(X \gamma) \gamma^{\prime}=X\left(\gamma \gamma^{\prime}\right)$ and $X 1=X$ for any $X \in C, \gamma, \gamma^{\prime} \in \bar{\Gamma}$ and 1 denoting the unit element in $\Gamma$;

$$
(f, F) \in \mathscr{V} \Rightarrow F(X \gamma)=F(X) \gamma \text { for each } X \in C \text { and } \gamma \in \bar{\Gamma} \text {. }
$$

Note that if $\bar{\Gamma}$ operates on $\mathscr{V}$ then $\Gamma$ becomes a Lie group of right transformations in each $C$. If $\mathscr{T}$ is a local semi-regular connector into $\mathscr{V}$ we shall say that it is $\bar{\Gamma}$-related if $\iota x \gamma=\iota x$ for each $x \in B, \gamma \in \bar{\Gamma}$ and $J_{1,2}\left(Z, Z^{\prime}\right) \gamma=J_{1,2}\left(Z \gamma \cdot Z^{\prime} \gamma\right)$ for each pair $B_{1}, B_{2}$ of differentiable manifolds and each $\left(Z, Z^{\prime}\right) \in \mathscr{T}\left(B_{1}\right) \times \mathscr{T}\left(B_{2}\right), \gamma \in \vec{\Gamma}$. This implies immediately that if $\left(Z, Z^{\prime}\right) \rightarrow Z . Z^{\prime}$ is the $\mathscr{T}$-prolongation of a composition law then $\left(Z . Z^{\prime}\right) \gamma=Z \gamma . Z^{\prime} \gamma$.

Point (3) of the above definition shows that each morphism $(f, F):(B, C) \rightarrow$ $\rightarrow\left(B^{\prime}, C^{\prime}\right)$ which belongs to $\mathscr{V}$ induces a morphism

$$
\left(f, F_{\Gamma}\right):(B, C / \Gamma) \rightarrow\left(B^{\prime}, C^{\prime} \mid \Gamma\right)
$$


of the right $\Gamma$-cosets, endowed with the natural coset differentiable structure, which belongs to $\mathscr{E}$.

Definition 3.8. Let $(B, C) \in \mathscr{V}$. An element $X \in C$ is called regular if $X \gamma=X \gamma^{\prime}$ for $\gamma, \gamma^{\prime} \in \bar{\Gamma}$ induces $\gamma=\gamma^{\prime}$. An element $X \in C$ is called incident with an element $X^{\prime} \in C$ if there exists $\gamma \in \bar{\Gamma}$ such that $X=X^{\prime} \gamma$.

Denote by $C^{*} \subset C$ the subset of regular elements in $C$. Each injective morphism of $\mathscr{V}$ takes regular elements into regular ones, and if a coset in $C / \Gamma$ contains a regular element then all its elements are regular. Such a coset is then also called regular.

Definition 3.9. An object $(B, C)$ of $\mathscr{V}$ is called $\bar{\Gamma}$-simple if there exists in each fibre of $(B, C)$ exactly one regular coset such that all the elements of this fibre are incident with some (and consequently with each) element of this coset.

Definition 3.10. A category $\mathscr{V}$ on which the semigroup $\bar{\Gamma}$ operates is called $\bar{\Gamma}$ complete if for each $(f, F):(B, C) \rightarrow\left(B^{\prime}, C^{\prime}\right)$ from $\mathscr{E}$ with $(B, C),\left(B^{\prime}, C^{\prime}\right)$ belonging to $\mathscr{V},(B, C)$ being $\bar{\Gamma}$-simple and $F$ satisfying $F(X \gamma)=F(X) \gamma, \gamma \in \bar{\Gamma}$ we have $(f, F) \in$ $\in \mathscr{V}$.

Finally note that if $\mathscr{T}$ is $\bar{\Gamma}$-related then $\mathscr{T}(\Phi)^{+}$being defined as above is invariant under the action of $\bar{\Gamma}$. Especially one can define $\mathscr{T}(\Phi)^{+} \mid \Gamma \subset \mathscr{T}(\Phi) / \Gamma$ and regular elements or cosets in $\mathscr{T}(\Phi)^{+}$.

Theorem 3.6. Let $\mathscr{T}$ be a $\bar{\Gamma}$-related semi-regular local connector into a $\bar{\Gamma}$-complete category $\mathscr{V}$. If $(B, \mathscr{T}(B))$ is $\bar{\Gamma}$-simple then a $(\mathscr{V}, \mathscr{T})$-connection on a fibre bundle $E\left(B, F, G^{*}, H, \Phi^{*}\right)$ can be uniquely characterized by a differentiable section $\mathscr{X}_{\Gamma}$ in $\left(B, \mathscr{T}(\Phi)^{+} \mid \Gamma\right)$ such that $\mathscr{T}(b) \mathscr{X}_{\Gamma}=\mathscr{T}(B)^{*} / \Gamma$.

Proof. Let $\mathscr{X}: \mathscr{T}(B) \rightarrow \mathscr{T}(\Phi)$ be a $(\mathscr{V}, \mathscr{T})$-connection on $E$ and define $\mathscr{X}_{\Gamma}(z)=$ $=\mathscr{X}\left(X_{z}\right)$, where $X_{z}$ is any regular element in $\mathscr{T}(B)_{z}$. This definition is clearly independent of the choice of $X_{z}$ and defines a differentiable section in $\left(B, \mathscr{T}(\Phi)^{+} / \Gamma\right)$.

Conversely let $\mathscr{X}_{\Gamma}$ be a differentiable section in $\left(B, \mathscr{T}(\Phi)^{+} / \Gamma\right)$. We shall define $\mathscr{X}$ for each element of $\mathscr{T}(B)^{*}$. Thus let $Z \in \mathscr{T}(B)^{*}$. There exists exactly one element $X=\mathscr{X}(Z)$ in the coset $\mathscr{X}_{\Gamma}(p Z)$ such that $\mathscr{T}(b) X=Z$. In fact if $X^{\prime}$ is any element in the coset $\mathscr{X}_{\Gamma}(p Z)$ then $\mathscr{T}(b) X^{\prime}=Z \gamma$ for some $\gamma \in \Gamma$ since $\mathscr{T}(b) X^{\prime}$ belongs to the only regular coset in $\mathscr{T}(B)_{z}$. Now if we put $X=X^{\prime} \gamma^{-1}$ we find the required value of $\mathscr{X}$ at $Z$. Moreover we see immediately from the regularity of $Z$ that this value is uniquely determined by $Z$. Since $(B, \mathscr{T}(B))$ is $\bar{\Gamma}$-simple we can completely define $\mathscr{X}$ by $\mathscr{X}(Z \gamma)=\mathscr{X}(Z) \gamma$. If now $Z$ varies differentiably in a neighbourhood of $z \in B$, as well as the above defined $X^{\prime}$, we see from the local expressions that the same is true about $X$ and by means of the $\bar{\Gamma}$-completeness of $\mathscr{V}$ we get $(\sim, \mathscr{X}) \in \mathscr{V}$ which now means that it defines a $(\mathscr{V}, \mathscr{T})$-connection on $E$.

Theorem 3.7. Let $\mathscr{T}$ be a $\bar{\Gamma}$-related semi-regular local connector into a local 
$\bar{\Gamma}$-complete category $\mathscr{V}$. If $(B, \mathscr{T}(B))$ is $\bar{\Gamma}$-simple then a $(\mathscr{V}, \mathscr{T})$-connection on a fibre bundle $E\left(B, F, G^{*}, H, \Phi^{*}\right)$ can be uniquely characterized by a differentiable section $h \rightarrow N_{h}$ in $(H, \mathscr{T}(H) / \Gamma)$ with the properties

(1) $N_{h g}=N_{h} . \iota g$ for each $g \in G$;

(2) $\mathscr{T}(b) N_{h}=\mathscr{T}(B)^{*} / \Gamma$.

Note that (2) implies $N_{h} \in \mathscr{T}(H)^{*} / \Gamma$.

Proof. Let $h \rightarrow N_{h}$ be such a section and for each $h \in H$ define $\mathscr{T}(H)_{h}^{+}$as the set of all elements in $\mathscr{T}(H)_{h}$ which are incident with an element of $N_{h}$. Especially $N_{h} \subset$ $\subset \mathscr{T}(H)_{h}^{+}$. We shall show that the conditions of theorem 3.3 are satisfied. We have clearly $\mathscr{T}(b) \mathscr{T}(H)_{h}^{+}=\mathscr{T}(B)_{b h}$ since for a fixed $X \in N_{h}$ the $\mathscr{T}(H)_{h}^{+}$consists of exactly all the elements $X \gamma, \gamma \in \bar{\Gamma}$ and $\mathscr{T}(b) X$ is regular. Further $\mathscr{T}(H)_{h g}^{+}=\mathscr{T}(H)_{h}^{+}$. .$\iota \mathrm{g}$ follows from (1). Construct the $\left(\xi_{U}, \Xi_{U}\right)$ as follows. If $\{U\}$ is a sufficiently fine covering of $B$ by open sets we have for each $U$ a differentiable section $\xi_{U}$ in $(U, H)$. Further if $h \rightarrow X_{h}$ is a local differentiable section in $(H, \mathscr{T}(H))$ with a suitable domain such that $X_{h} \in N_{h}$, define for a fixed differentiable section $z \rightarrow Z$ in $\left(U, \mathscr{T}(U)^{*}\right)$ the local mapping $\Xi_{U}$ by $\Xi_{U}(Z \gamma)=X_{\xi_{U}(z)} \gamma$. Now it is not difficult to see that the $\left(\xi_{U}, \Xi_{U}\right)$ thus defined has all the properties required in theorem 3.3.

Conversely let there be given the subspaces $\mathscr{T}(H)_{h}^{+}$as in theorem 3.3. Let $N_{h}$ be the set of all regular elements of $\mathscr{T}(H)$ contained in $\mathscr{T}(H)_{h}^{+}$. They form a $\Gamma$-coset. In fact, there is a one-to-one mapping $\left.\Xi_{U}^{*}\right|_{b h}: \mathscr{T}(U)_{b h} \rightarrow \mathscr{T}(H)_{h}^{+}$defined in the proof of theorem 3.3 and such that $\left(\xi_{U}, \Xi_{U}^{*}\right)$ belongs to $\mathscr{V}$. Next we get easily $N_{h g}=N_{h} . \imath g$ and $\mathscr{T}(b) N_{h}=\mathscr{T}(B)^{*} / \Gamma$. Especially $N_{h}$ is not empty. It remains to show that $h \rightarrow N_{h}$ is differentiable. $U \subset B$ being given as above define the mapping $b^{-1}(U) \subset H \rightarrow$ $\rightarrow \mathscr{T}(H) \mid \Gamma$ by $h=\xi_{U}(x) \cdot g \rightarrow \pi_{\Gamma}\left(\Xi_{U}(Z(x)) \cdot \iota g\right)$, where $x \rightarrow Z(x)$ is any differentiable section in $\left(U, \mathscr{T}(U)^{*}\right)$ and $\pi_{\Gamma}$ is the natural projection $\mathscr{T}(H) \rightarrow \mathscr{T}(H) / \Gamma$. This is a differentiable mapping and assigns to $h$ the coset $N_{h}$. This completes the proof.

We shall apply these considerations to the category $\widetilde{C}_{s}^{r}\left(V_{p},.\right)$. If we denote by $\tilde{J}_{s}^{r}\left(V_{p}\right)$ the semigroup of all non-holonomic $r$-jets from $V_{p}$ into $V_{p}$ with source and target $s$, we get a semigroup of operators on this category and the connector $\tilde{J}_{s}^{r}\left(V_{p},.\right)$ is $\tilde{J}_{s}^{r}\left(V_{p}\right)$-related. It is not difficult to see that an element $X \in \widetilde{C}_{s}^{r}\left(V_{p}, B\right)$ is regular iff it is an injective jet and that $\left(B, \tilde{J}_{s}^{r}\left(V_{p}, B\right)\right)$ is $\tilde{J}_{s}^{r}\left(V_{p}\right)$-simple iff $\operatorname{dim} V_{p}=\operatorname{dim} B$ and in this case an element $X$ is regular iff it is inversible. One sees that the category $\widetilde{C}_{s}^{r}\left(V_{p},.\right)$ is also $\tilde{J}_{s}^{r}\left(V_{p}\right)$-complete. In fact let $(f, F)$ be given as in definition 3.10. If $z \in B$ is fixed and $X \in \tilde{J}_{s}^{r}\left(V_{p}, B\right)_{z}$ is inversible then there exists exactly one $Z \in \tilde{J}_{s}^{r}\left(B, B^{\prime}\right), \alpha(Z)=z$, $\beta(Z)=f(z)$ such that $F(X)=Z X$ and thus $F(X \gamma)=Z X \gamma$. Since $X \gamma \rightarrow F(X \gamma)$ is differentiable, one sees also that $z \rightarrow Z$ is differentiable, i.e. $(f, F) \in \tilde{J}_{s}^{r}\left(V_{p},.\right)$. The elements of $\tilde{J}_{0}^{r}\left(R^{k}, B\right)^{*} / \tilde{J}_{0}^{r}\left(R^{k}\right)=\left(\widetilde{T}_{k}^{r}\right)^{*} / \tilde{L}_{k}^{r}$ are the usual non-holonomic $k^{r}$-contact elements of the manifold $B$ (cf. [4]).

The situation is analogous in the semi-holonomic and hoionomic cases. Note however that e.g. $J_{s}^{r}\left(V_{p}\right)$ is also a semigroup of operators on $\tilde{J}_{s}^{r}\left(V_{p},.\right)$ but $\operatorname{dim} V_{p}=$ 
$=\operatorname{dim} B \neq 0$ does not imply that $\left(B, \tilde{J}_{s}^{r}\left(V_{p}, B\right)\right)$ is $J_{s}^{r}\left(V_{p}\right)$-simple, moreover, there do not exist in general $J_{s}^{r}\left(V_{p}\right)$-simple (or $\bar{J}_{s}^{r}\left(V_{p}\right)$-simple) objects in the category $\tilde{J}_{s}^{r}\left(V_{p},.\right)$. Especially $\tilde{J}_{s}^{r}\left(V_{p},.\right)$ is trivially $J_{s}^{r}\left(V_{p}\right)$ - $\left(\right.$ or $\bar{J}_{s}^{r}\left(V_{p}\right)$-)complete.

If one takes in these special cases $V_{p}=R^{n}(n=\operatorname{dim} B)$ and $s=0$ one gets in theorem 3.6 the characterization of higher order connections by means of contact elements introduced by Ehresmann in [5] (cf. also our definition 2.3), and in theorem (3.7) that given in [1] and [2].

\section{References}

[1] B. Cenkl: On the higher order connections, Cahiers de Topologie et Géom. Diff., Vol. IX (1967), 11-32.

[2] B. Cenkl: Infinitesimal connections of higher order, Revue Roumaine Math. Pures et Appl., Tome $X(1965), 1277-1280$.

[3] Ch. Ehresmann: Les connexions infinitésimales dans un espace fibré différentiable, Colloque de topologie, Bruxelles 1950, 29-55.

[4] Ch. Ehresmann: Les prolongements d'une variété différentiable, C. R. Acad. Sci. Paris 233 (1951), 598-600, 777-779, 1081-1083; 239 (1954), 1762-1764; 240 (1955), 397-399, $1755-1757$.

[5] Ch. Ehresmann: Sur les connexions d'ordre supérieur, Atti del V. Congresso dell'Unione Mat. Ital. Pavia-Torino, 1956, 1-3.

[6] Ch. Ehresmann: Gattungen von lokalen Strukturen, Jahresbericht Deutsch. Math. Ver., Bd. 60 (1957), 49-77.

[7] Ch. Ehresmann: Catégories topologiques et catégories différentiables, Coll. Géom. Diff. Glob., Bruxelles, 1959.

[8] Ch. Ehresmann: Prolongements des catégories différentiables, Topologie et Géom. Diff., Vol. VI (1964), $1-8$.

[9] E. Feldmann: The geometry of immersions, Bull. Amer. Math. Soc., 69 (1963), 693-698.

[10] P. Libermann: Connexions d'ordre supérieur, $3^{\circ}$ Coloquio Brasileiro de Mat. Fortaleza, 1961.

[11] P. Libermann: Calcul tensoriel et connexions d'ordre supérieur, Anais da Acad. Bras. de Ciencias, 37 (1965), 17-29.

[12] P. Libermann: Sur la géometrie des prolongements des espaces fibrés vectoriels, Ann. Inst. Fourier, Grenoble, 14 (1964), 145-172.

[13] P. Libermann: Surconnexions, C. R. Acad. Sci. Paris, 258 (1964), 6327-6330; 261 (1965), $2801-2804$.

[14] K. Nomizu: Lie Groups and Differential Geometry, Publ. Math. Soc. Japan 2 (1956).

[15] W. Pohl: Connexions in differential geometry of higher order, (polycopied notes), Stanford University, 1963.

[16] W. Pohl: Connexions in differential geometry of higher order, Transactions Amer. Math. Soc., 125 (1966), 310-325.

[17] P. Ver Eecke: Sur les connexions d'éléments de contact, Thèses, Topologie et Géom. Diff., 1962.

[18] J. Virsik: Non-holonomic connections on vector bundles, Czech. Math. J. 17 (1967), I : $108-147 ;$ II : 200-224.

[19] J. Virsik: Über Zusammenhänge höherer Ordnungen in Vektorraumbündeln, Math. Nachrichten 34 (1967), $219-227$.

Author's address: Bratislava, Štefánikova 41, ČSSR (Matematický ústav SAV). 